\title{
The Siren Song of Implicit Change Detection
}

\author{
Stephen R. Mitroff, Daniel J. Simons, and Steven L. Franconeri \\ Harvard University
}

\begin{abstract}
Although change blindness could suggest that observers represent far less of their visual world than their conscious experience leads them to believe, they could fail to detect changes even if they fully represent all details. Reports of implicit change detection in the absence of awareness are consistent with the notion that observers' representations are more complete than previously thought. However, to provide convincing evidence, studies must separate implicit detection from explicit processes. This article reexamines the 3 primary claims of implicit change detection and, after replicating original findings, provides theoretical and empirical support for alternative, explicit explanations. Even if observers do represent more of the scene than previously thought, change detection might occur only through explicit comparisons.
\end{abstract}

Most people are comfortable with the notion that perception can occur without awareness, and the perception and cognition literatures have seen repeated claims for implicit perception (e.g., see Dixon, 1981). Most attempts to demonstrate the existence of implicit perception have relied on the dissociation paradigm in which evidence that observers lack explicit awareness of a stimulus is contrasted with evidence for the effects of that stimulus on performance. However, for such evidence of implicit detection to be convincing, the experimental design must meet a number of stringent criteria. Recent methodological and theoretical critiques raise doubts about whether these criteria have been met (Holender, 1986). The main difficulty in proving the existence of implicit perception resides in demonstrating the complete absence of awareness. For example, evidence for semantic processing without awareness (i.e., implicit perception of meaning) requires both positive evidence for a behavioral effect of the critical stimulus and definitive evidence that the stimulus did not reach awareness when it was initially presented (Holender, 1986). If the stimulus was above the perceptual threshold for detection, then it is always possible that observers might have explicitly detected it. Consequently, the most convincing evidence for implicit perception

Stephen R. Mitroff, Daniel J. Simons, and Steven L. Franconeri, Department of Psychology, Harvard University.

Stephen R. Mitroff was supported by a National Science Foundation (NSF) graduate fellowship, and Daniel J. Simons was supported by NSF Grant BCS-9905578 and by a fellowship from the Alfred P. Sloan Foundation.

We thank Eileen Bent, Eric Black, Michael Brusco, Erin Clifford, Cendri Hutcherson, Sue Paik, and Joseph Vuckovich for their assistance in data collection. The arguments in this article benefited from helpful debates and discussions with Marvin Chun, Don Dulany, Andrew Hollingworth, Brian Scholl, Daniel Smilek, Ian Thornton, and Patrick Wilken. We also thank Diego Fernandez-Duque, Eyal Reingold, Ron Rensink, and an anonymous reviewer for thoughtful reviews of an earlier version.

Correspondence concerning this article should be addressed to Stephen R. Mitroff, who is now at Department of Psychology, Yale University, Kirtland Hall, P.O. Box 208205, New Haven, Connecticut 06520-8205, or to Daniel J. Simons, who is now at Department of Psychology, University of Illinois at Urbana-Champaign, 603 East Daniel Street, Champaign, Illinois 61820. E-mail: Stephen.Mitroff@yale.edu or dsimons@uiuc.edu requires that the critical stimulus be below threshold at the time of the initial presentation (Holender, 1986).

Stated more precisely, convincing evidence for implicit perception in the dissociation paradigm requires that the task meet three specific criteria (Merikle \& Reingold, 1998; Reingold \& Merikle, 1988). First, the measure of explicit awareness must assess all consciously available information (the exhaustiveness assumption). If a measure assesses only some components of explicit awareness, then any evidence for implicit perception could be tainted by unmeasured explicit components. Second, to demonstrate that a measure is not tainted by explicit awareness, the exhaustive explicit measure must reveal no sensitivity to the critical stimulus. That is, observers must not have explicit awareness of any sort. Finally, the implicit measure must demonstrate some sensitivity to the critical stimulus. If all three of these criteria are met for the same displays, then evidence for implicit detection is unambiguous. Essentially, if no explicit measure could reveal awareness but performance is still affected by a critical stimulus, then perception must have been implicit. When these stringent criteria are applied to evidence for implicit perception in the dissociation paradigm, few if any studies provide unequivocal evidence for perception without awareness (Holender, 1986; Merikle \& Reingold, 1998; Reingold \& Merikle, 1988).

This issue of how to distinguish implicit from explicit processing has plagued many literatures. Claims of implicit memory or implicit processing are often countered by claims of explicit contamination (e.g., see Butler \& Berry, 2001, and Ratcliff \& McKoon, 1995, for critiques of work on implicit memory). Recently, the literature on change detection has seen a number of claims for implicit detection in the absence of awareness. The study of change detection has produced striking evidence that observers often fail to notice large changes to scenes when the changes are contingent on a visual disruption, a phenomenon known as change blindness (for reviews, see Rensink, 2000a; Simons, 2000; Simons \& Levin, 1997). Although observers do not report noticing changes, they might still have detected these changes without awareness. The stringent criteria just discussed provide a framework for examining recent claims of implicit change detection. Note that, in this article, we examine claims of implicit change detection but not implicit perception more generally. Change detec- 
tion requires observers to compare a representation of the prechange information to the postchange information. That is, successful change detection requires observers to both form a representation and compare that representation. Thus, claims of implicit change detection require not just the implicit detection of the presence of a stimulus but also an implicit comparison process. Implicit perception does not necessarily require such a comparison.

In this article, we replicate several of the findings that underlie recent claims of implicit change detection and then show, both theoretically and empirically, how an explicit explanation is equally or more plausible. In essence, we show that these findings do not meet the exhaustiveness assumption by demonstrating that explicit awareness could produce the same results. Evidence for implicit change detection cannot be convincing unless it can be shown to be devoid of explicit influences (Reingold \& Merikle, 1988). The broad goal of this article is to sound a cautionary note against premature claims of implicit change detection by noting how explicit factors can influence performance even when observers do not report noticing changes. We draw on previous theoretical arguments and methodological critiques to suggest guidelines for future attempts to demonstrate implicit change detection, and we provide some speculative arguments that implicit change detection might not be possible in principle. As with any strong theoretical claim, our position is refutable by solid empirical evidence, and we provide suggestions for approaches that could disconfirm our thesis.

\section{EVIDENCE FOR CHANGE BLINDNESS}

Change blindness occurs in a wide variety of tasks and under a wide array of viewing conditions, provided the change coincides with a disruption or distraction. Change blindness for unexpected changes has been found when changes occur during camera cuts or pans in motion pictures (Levin \& Simons, 1997; Simons, 1996) and during real-world disruptions (Simons \& Levin, 1998). When observers actively search for changes, they often fail to detect them if they coincide with an eye movement (Grimes, 1996; Henderson \& Hollingworth, 1999), a blink (O’Regan, Deubel, Clark, \& Rensink, 1997), a flashed blank screen (Pashler, 1988; Rensink, O'Regan, \& Clark, 1997), or some other visual distraction (O’Regan, Rensink, \& Clark, 1999; Rensink, O’Regan, \& Clark, 2000). When an original and a modified display alternate rapidly and repeatedly, separated by a blank screen (the flicker task), observers often take many seconds to detect even large changes (Rensink et al., 1997). In all of these cases, change detection is difficult because the visual disruption hides or masks the change signal that would otherwise accompany the change (Rensink et al., 1997). Without a visual disruption, changes will produce a signal that can draw attention (but see Simons, Franconeri, \& Reimer, 2000, for evidence that disruptions are not always necessary to produce change blindness).

Without a change signal to draw attention, detection must occur through an inference process (James, 1891/1950). Observers must initially form a representation, or create a memory trace, of the first display. Then the representation must be compared to the second display. Next, observers must infer from the comparison that something changed from the first to the second display. The need for such a comparison process can be simulated by placing an original and a changed image next to each other and trying to find the change (Scott-Brown, Baker, \& Orbach, 2000; Shore \& Klein,
2000). The difficulty in spotting the difference between these images is comparable to the difficulty of detecting changes across a visual disruption. In both cases, the difficulty of detection suggests that little information is explicitly retained and compared from one view to the next. That is, if observers were able to fully represent any given scene such that it could subsequently be compared to the changed scene, any change should be readily noticed.

Although change detection is often quite difficult, failure to detect a change does not imply the absence of a representation or a failure to compare representations (Simons, 2000). Typically, change blindness studies require an explicit report of detection. Knowledge of whether or not a change occurred might be too conservative a measure of detection and might drastically underestimate the amount of information that is represented and/or compared across views. Some recent evidence from change detection studies using motion pictures and real-world events seems to support this conclusion. In one study (Simons, Chabris, Schnur, \& Levin, 2002), an experimenter who was holding a basketball approached a pedestrian to ask for directions to a gymnasium. While the pedestrian provided directions, a group of people walking down the sidewalk passed between the pedestrian and experimenter, and one member of the group surreptitiously removed the basketball. When the pedestrian finished giving directions, the pedestrian was asked if he or she had noticed anything unexpected happen or if he or she had noticed a change. Most did not spontaneously report the change. However, when asked further leading questions, many reported the presence of the basketball and were even able to describe its features. They did not notice the change (i.e., they were change blind), but they still had represented details of the changed object.

In a related series of studies, observers viewed a videotape of a similar interaction in which clothing or objects were changed during a cut in camera position (Angelone, Levin, \& Simons, 2001). Even those observers who did not report the change spontaneously were able to select the prechange appearance of the actor from a photographic lineup at better-than-chance levels. Both of these findings might provide evidence for preserved implicit representations in the face of change blindness, but that possibility was not directly tested. Evidence from a number of literatures further suggests that observers may retain a representation of a scene without awareness (e.g., Chun \& Jiang, 1998; Chun \& Nakayama, 2000; Maljkovic \& Nakayama, 1994, 1996). Moreover, these representations may directly influence behavior. For example, participants find a target more efficiently when they have seen the search array before, even if they do not recognize the display (Chun \& Jiang, 1998). However, none of these findings provide any evidence for implicit change detection. Just because observers might have implicitly represented a changed part of a scene does not mean that they implicitly detected the change itself. By definition, change detection requires a comparison process and not just a representation.

Despite this caveat, the presence of preserved representations in the face of change blindness raises the possibility that the change might be detected without awareness. If observers have a representation (either implicit or explicit) of the prechange scene, then, in principle, an implicit comparison mechanism could produce detection without awareness. If so, then more sensitive measures of change detection might reveal the presence of implicit detection without awareness. Explicit reports of change detection might 
underestimate actual change detection because they neglect the possibility of implicit representations and an implicit comparison mechanism. This possibility has inspired the search for evidence of change detection in the absence of awareness.

\section{IMPLICIT CHANGE DETECTION}

Empirical claims of evidence for implicit change detection can be categorized into three distinct classes: registration, localization, and identification. The most conservative claims for implicit change detection posit only that changes are implicitly registered. A change is implicitly registered if it somehow influences performance, regardless of whether or not it influences explicit detection. Any behavioral evidence that differentiates the presence of a change from the absence of a change without awareness of the change constitutes evidence for implicit registration (e.g., Williams \& Simons, 2000). For example, change registration could include slowed response times in the presence of an unnoticed change or longer looking times in the presence of an unnoticed change than in the absence of a change. The key to evidence for implicit registration is that the change was not explicitly detected or registered in any way. That is, the change exerts some influence even though it was not consciously detected. In contrast to registration, implicit localization requires that observers be unaware of the change and that they localize it better than would be expected by chance (Fernandez-Duque \& Thornton, 2000). Some claims of implicit change localization suggest that, before awareness of a change, implicit detection can guide attention to the change, thereby allowing explicit localization (Smilek, Eastwood, \& Merikle, 2000). Evidence for implicit localization requires changes to be implicitly registered and localized without explicit detection or localization. Finally, a change is implicitly identified if the nature or identity of the change can influence processing in the absence of awareness of the change itself (Thornton \& FernandezDuque, 2000). Note that implicit identification requires registration along with some additional processing of the change itself but does not necessarily require localization of the change.

In this article, we examine each of these classes of implicit change detection in turn. For each, we first present the evidence used to support claims of implicit change detection and then, using both thought experiments and new empirical work, we show that explicit mechanisms can also account for the effects. Although the existing work might suggest the presence of implicit change detection, our new findings offer alternative explanations that do not necessarily rely on implicit detection. For several of these cases, our evidence reveals a failure to meet the exhaustiveness assumption, which is a necessary criterion for the demonstration of implicit perception in the dissociation paradigm. We offer four separate sets of experiments, each addressing recently published claims of implicit detection. Experiment 1 explored the evidence for implicit registration. Experiments 2 and 3 examined implicit localization. Experiment 4 examined implicit identification.

\section{EXPERIMENT 1: IMPLICIT REGISTRATION}

The primary evidence for implicit change registration comes from a difference in response times when observers fail to notice a change that is present in a scene and when there is no change present in the scene. In one study, a single object was presented, removed, and then presented again either with nothing changed or with one, two, or three parts of the object changed (Williams \& Simons, 2000). Observers responded as quickly as possible whether the two presentations were the same or different. To demonstrate implicit registration, an experiment must reveal an effect of the presence of a change on performance even when observers are unaware of the change. By responding same, observers indicated that they were unaware of any change. Therefore, the critical comparison was between erroneous same responses on trials with a change and correct same responses on trials with no change. The results showed that $68 \%$ of observers were quicker to respond same when there was no change than when there was a change. That is, when observers reported no awareness of the change by responding same, their response latency differed depending on whether or not a change occurred. This finding of an effect on response latency was taken to imply implicit change detection.

However, this experiment only minimally controlled for explicit awareness of the change. Observers might respond same with varying degrees of confidence depending on their criterion. In general, observers were biased to respond same, suggesting that they adopted a relatively conservative criterion for different responses. Observers reported different only when they were certain of their detection, and they might have responded same in some cases in which they thought a change had been present but were not certain. In the terminology of the dissociation paradigm, the use of same responses as an indication of the absence of awareness might not provide an exhaustive measure of the absence of awareness (Reingold \& Merikle, 1988). Given that confidence is often correlated with response latency, erroneous same responses might be slower as a result of reduced confidence in explicit detection rather than implicit detection. This uncertainty, combined with a bias to respond same, would produce slower response times for erroneous same responses even in the absence of implicit detection.

In this experiment, we examined whether or not confidence underlies the response time difference by first attempting to replicate the critical finding of a response time difference and then by correlating this difference with a measure of confidence. Observers viewed a display of two radial color gratings (see Figure 1) followed by a blank screen and then by another display of two gratings. On half of the trials the second display was identical to the first, and on the other half one of the two gratings was rotated in the picture plane. Observers reported whether the two displays were the same or different and then rated their confidence in their response. If changes are implicitly registered, confidence should not mediate or account for the relationship between response latency and the presence or absence of a change. Alternatively, if confidence accounts for the relationship, then the evidence for implicit change registration is equivocal.

\section{Method}

Participants. Twenty undergraduates received course credit for their participation.

Materials. Displays were presented on an iMac computer with a 15-in. $(38-\mathrm{cm})$ monitor set to a resolution of $1,024 \times 768$ and a refresh rate of 75 $\mathrm{Hz}$. Observers sat at a comfortable distance from the monitor without head restraint. All stimulus presentations and data collection were accomplished through custom software written with Vision Shell C libraries (http://www.visionshell.com). 
A

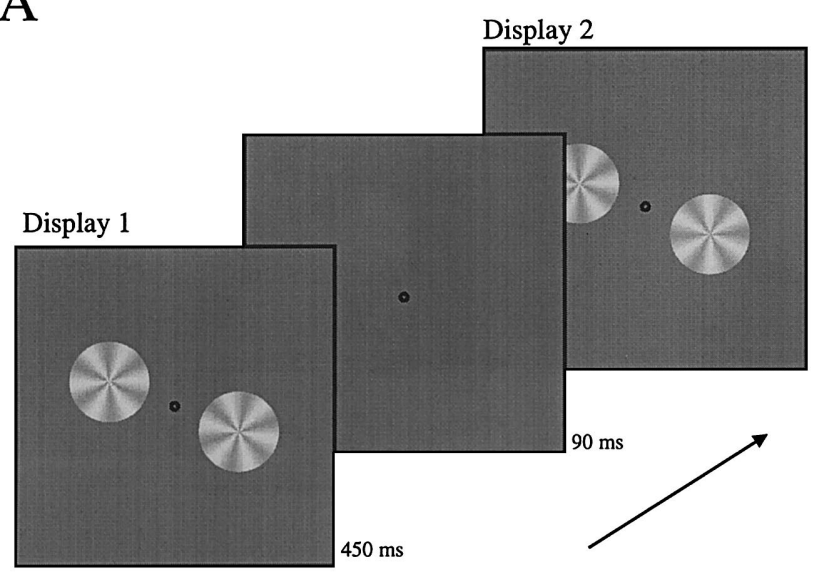

$\mathrm{C}$

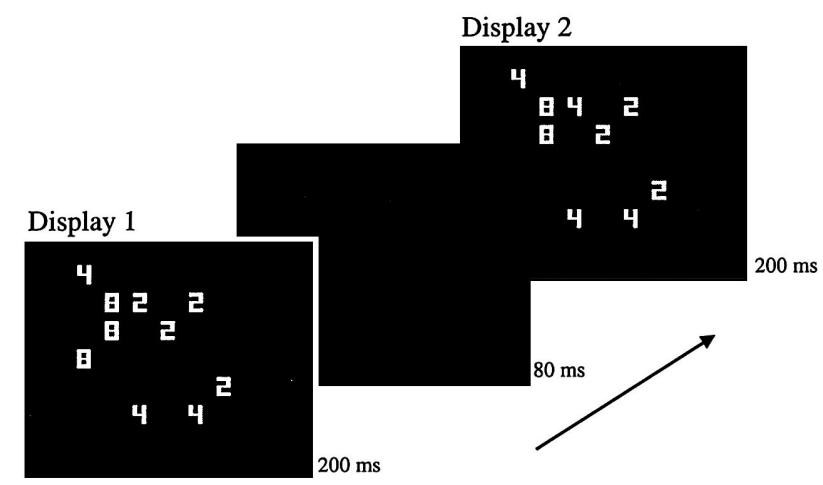

B

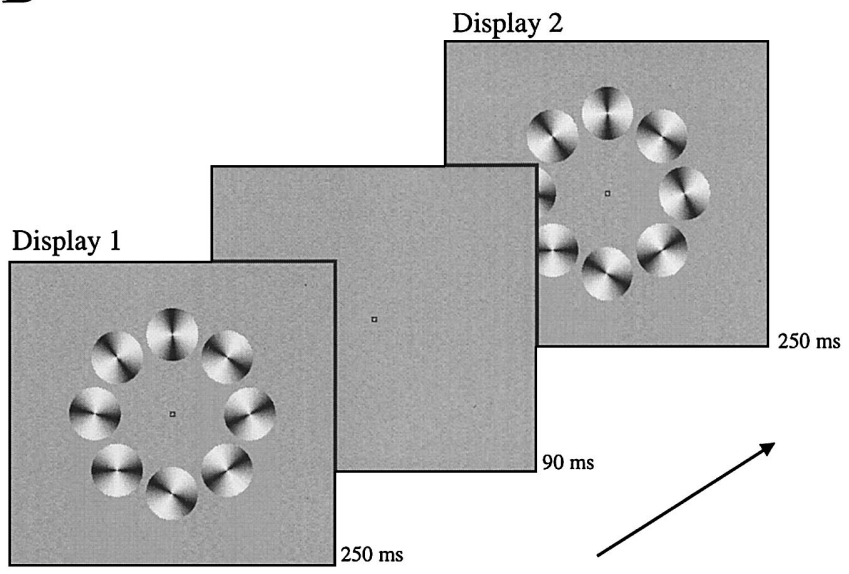

$\mathrm{D}$

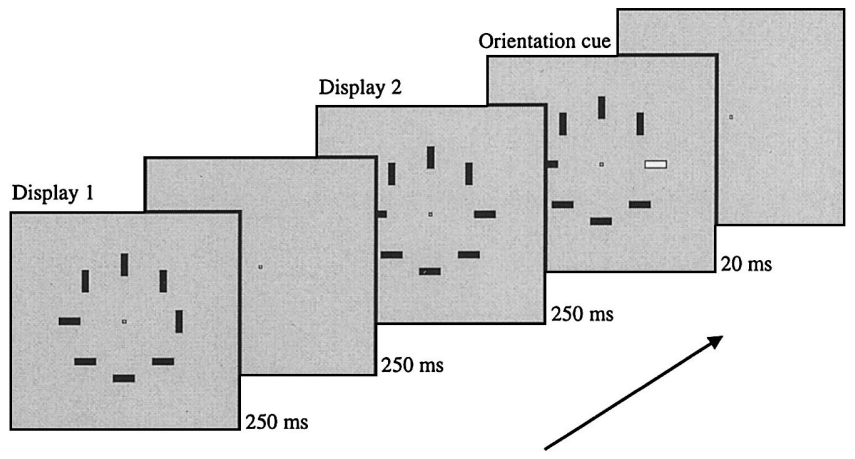

Figure 1. Stimuli and timings used in each experiment. A: Displays used in Experiment 1 consisted of two equiluminant red and green sinusoidally varied radial gratings composed of six cycles. Here the stimuli are shown as a luminance grating against a different background for illustrative purposes. Changes were induced by rotating one of the gratings. In this example, the object on the right was changed. B: Displays used in Experiment 2 consisted of eight equiluminant red and green sinusoidally varied radial gratings composed of two cycles. Changes were induced by rotating one of the gratings. The right-most item was changed in this example. C: Displays used in Experiment 3 consisted of 4, 7, 10, 13, or 16 digits randomly assigned to locations within an imaginary $6 \times 6$ grid. In Experiments 3A and 3B the item locations were held constant throughout a single trial, whereas in Experiment $3 \mathrm{C}$ the item locations were randomly reassigned on each cycle of the trial. In Experiment 3A, the change was repeatedly cycled (a flicker task). In Experiments 3B and 3C, observers responded after each discrete cycle (the final display remained visible). D: Experiment 4 involved an initial display of four horizontal and four vertical rectangles, and on $80 \%$ of the trials one of the rectangles changed orientation in the second display. Shortly after the second array appeared, one item was cued by a color change for $20 \mathrm{~ms}$, and then the array was removed. In Experiment 4A, the cued item was always the changed item or the diametrically opposite item. In Experiment 4B, there was no relationship between the cued and changed locations.

All displays consisted of a gray background $\left(10 \mathrm{~cd} / \mathrm{m}^{2}\right)$ with two radial six-cycle color gratings on opposite sides of a central fixation point. Although the gratings always were on diametrically opposite sides of the fixation point, their orientation relative to the display was randomized on each trial. Assuming an average viewing distance of $50 \mathrm{~cm}$, the centers of the two gratings were approximately $11^{\circ}$ apart. Each grating subtended $6.36^{\circ}$ and varied sinusoidally in color saturation, from a maximum green of $20.2 \mathrm{~cd} / \mathrm{m}^{2}$ to a maximum red of $16.2 \mathrm{~cd} / \mathrm{m}^{2}$ with a mean luminance of 18.2 $\mathrm{cd} / \mathrm{m}^{2}$. The green values were made sufficiently bright to account for the difference in perceptual luminance between red and green (Cavanagh, MacLeod, \& Anstis, 1987). The initial phase of each grating was random- ized before each trial, and on change trials one of the two gratings underwent a $90^{\circ}$ phase shift. The colors in the gratings were made to be approximately perceptually equiluminant to reduce the luminance-based motion signal produced by the phase shift.

Procedure. Participants completed 20 practice trials before completing 384 individually randomized test trials. They were instructed to rest whenever they desired and were prompted to take a brief rest after the 192nd trial. Each trial was initiated when observers pressed a key. A fixation point then appeared at the center of the display, remaining visible throughout the trial. Two blue outline circles (1-pixel line width) appeared in the positions that would subsequently be occupied by the gratings, and after $1,500 \mathrm{~ms}$ 
the cues were replaced by the initial display of two radial color gratings. The gratings were presented for $450 \mathrm{~ms}$, followed by a full screen pattern mask consisting of red and green noise for $90 \mathrm{~ms}$ and then by the second display of two gratings in the same location. The second display remained visible until participants responded. On half of the trials, one of the two radial gratings was rotated in the picture plane relative to the first display.

On each trial, observers first reported whether the two displays were the same or different using the $S$ or $D$ key, respectively. After a 500-ms delay, they were prompted to rate their confidence in their response on a 1 to 5 scale according to the following criteria:

1-The same/different response was arbitrary. You responded "same" or "different" only because you were forced to respond, not because you felt one way or the other.

2-You had some reason for saying either "same" or "different." That is, you purposely responded "same" instead of "different" (or vice versa), yet you were very unsure of the accuracy of the response.

3-You had a reason for responding "same" or "different" but there was still some room for doubt.

4 -You are fairly certain that your response was correct, but not $100 \%$ certain.

5-You are absolutely certain of the accuracy of your response. You saw the change and responded "different" or you definitely saw that there was no change and responded "same."

\section{Results and Discussion}

Before any analysis of the data, outlying response times were removed for each observer. Any response time more than four standard deviations away from the mean response time for a given observer was eliminated from further analyses. On average, $1.86 \%$ of the data points were eliminated per observer $(S D=1.24 \%)$.

Overall, observers noticed $34.67 \%$ of the changes (mean false alarm rate: $15.21 \%$ ). According to a signal detection analysis, ${ }^{1}$ sensitivity to the changes was quite high (mean $\left.A^{\prime}=.819\right)$, and observers were slightly biased to respond same (mean $\left.B^{\prime \prime}=.108\right)$. Consistent with earlier findings (Williams \& Simons, 2000), observers were quicker to reply same when there was no change $(M=937 \mathrm{~ms})$ than when there was a change $(M=1,040 \mathrm{~ms})$, $t(19)=4.09, p<.001$. This pattern held for $80 \%$ of the observers. As in the original studies (Williams \& Simons, 2000), the magnitude of the response time difference between correct and incorrect same trials for each individual was not correlated with bias $(r=$ $.177, p=.456$ ). The difference in response times, however, was significantly correlated with sensitivity $(r=.741, p=.001)$ such that participants who were more sensitive to the presence of a change exhibited a larger difference in response times. It is not clear that this relationship has any bearing on claims of implicit change detection.

As predicted, observers responded more rapidly when they were more confident (mean $r=.282$ ), ${ }^{2}$ supporting the possibility that response time differences might result from differences in confidence rather than from implicit detection. Consistent with this possibility, observers were, on average, less confident when they incorrectly responded same $(M=3.19$ out of 5) than when they correctly responded same (3.87 out of 5), $t(19)=6.03, p<.001$. Across observers, larger differences between mean response times for correct and incorrect same responses were associated with larger differences in confidence $(r=.506, p=.023)$. To determine whether confidence or implicit detection was primarily responsible for the difference in response times, we regressed each participant's confidence and the presence-absence of change on the response time for all trials on which he or she responded same.
Confidence alone accounted for $29.24 \%$ of the response time variability in same responses, whereas the presence-absence of a change alone accounted for only $15.07 \%$. Furthermore, after accounting for the contribution of confidence to response times, the presence-absence of a change accounted for only an additional $2.41 \%$ of the response time variability. In contrast, after accounting for the contribution of the presence-absence of a change, confidence still accounted for $16.58 \%$ of the variability. On the basis of this analysis, confidence appears to better account for the difference in response times than does the presence or absence of a change, suggesting that the response time difference might not have resulted from implicit change detection.

Of course, one could argue that confidence ratings are based on implicit detection, and the presence or absence of an implicitly detected change mediates confidence. However, given the overall bias to respond same, it seems reasonable to conclude that observers explicitly shift their criterion to be conservative when detecting changes. This conservative bias might well result in some trials for which observers explicitly detected something changing but were not sufficiently confident to respond different. Although an implicit interpretation of this confidence effect is certainly possible, these findings raise doubts about the implicit detection interpretation. Given that confidence might well be based on explicit detection, these data do not provide sufficient evidence to conclude that the change was implicitly registered.

In addition to this claim for implicit registration, several other findings suggesting the possibility of implicit change registration deserve comment. Across a number of experiments in which changes are made to displays during saccades, observers appear to look longer at the changed object even when they did not consciously detect the change (Hayhoe, Bensinger, \& Ballard, 1998; Henderson \& Hollingworth, 1999; Hollingworth, Williams, \& Henderson, 2001). In one paradigm, observers study a static image (often for an extended duration) for a later memory test, and changes are sometimes introduced during a saccade. Observers are told to report any changes they see. Under these conditions, when observers fail to detect a change, on refixation of the changed object, they tend to look longer than if no change had occurred (Henderson \& Hollingworth, 1999; Hollingworth et al., 2001). This finding might suggest that the change was detected but did not surpass a threshold for awareness. That it still influenced fixations might then be taken as evidence for implicit detection. However, as in the response time case, there is no way to guarantee that observers truly were unaware of the change when it was missed (Hollingworth et al., 2001). Without a more exhaustive

\footnotetext{
${ }^{1}$ Here we chose to use nonparametric indexes of discrimination $\left(A^{\prime}\right)$ and bias $\left(B^{\prime \prime}\right)$, which are less subject to deviations from a normal distribution (Snodgrass \& Corwin, 1988). $A^{\prime}$ varies from 0.0 to 1.0 , with .5 representing chance performance. $B^{\prime \prime}$ varies from -1.0 to 1.0 , with 0 indicating no bias. When observers attempt to maximize hits at the cost of false alarms, $B^{\prime \prime}$ will be negative, and when observers minimize false alarms at the cost of fewer hits, $B^{\prime \prime}$ will be positive. $A^{\prime}$ and $B^{\prime \prime}$ are defined such that when the hit rate $(H)$ is greater than the false alarm rate $(F A), y=H$ and $x=F A$, and when $F A>H, y=F A$ and $x=H: A^{\prime}=.5+[(y-x)(1+y-x)] /[4 y(1-x)]$, and $B^{\prime \prime}=[y(1-y)-x(1-x)] /[y(1-y)+x(1-x)]$.

${ }^{2}$ We calculated all mean correlations reported in this article by first converting each correlation to a Fisher $r_{z}$ value to place it in a normal distribution, then taking the mean, and finally converting the mean back to the corresponding correlation value (Rosenthal \& Rosnow, 1991).
} 
measure of awareness than a verbal report of change detection, evidence for effects on response latencies or fixation durations does not provide convincing support for the existence of implicit change detection.

\section{EXPERIMENTS 2 AND 3: IMPLICIT LOCALIZATION}

Recall that evidence for implicit localization requires implicit registration of the presence of a change and some effect of the change on localization, both without explicit detection of the change. Two approaches have been used to examine implicit localization. The first, examined in Experiment 2, considers whether or not observers can guess the location of a change even when they report no awareness of having seen the change. The second, examined in Experiment 3, considers whether detection of the change without awareness leads to gradual attraction of attention to the change location. This attraction process might then lead to explicit localization and detection.

Fernandez-Duque and Thornton (2000) showed that when observers report being unaware that a change occurred and are then forced to guess between two potential change locations, they are more likely to select the actual change location than the unchanged location. Intuitively, this finding suggests that observers registered the location of the change implicitly even though they did not consciously perceive the change. In the Fernandez-Duque and Thornton study, a circular array of horizontally or vertically oriented rectangles was shown briefly and then removed. After a short delay, the array reappeared with one of the rectangles rotated by $90^{\circ}$. Two of the rectangles were then cued (the one that had changed and the diametrically opposite one), and observers were asked to select the one they thought had changed. Next, observers were asked to indicate whether or not they had seen the change. The critical finding came from the condition in which observers reported not seeing the change: When the change was undetected, observers still selected that changed item significantly more often than the nonchanged item (i.e., more than $50 \%$ of the time in a two-alternative forced-choice task).

In the second approach used to demonstrate implicit change localization (addressed in Experiment 3), Smilek et al. (2000) considered whether or not information about the change location accumulates implicitly before explicit detection. Their studies involved a flicker task in which the change occurred repeatedly during a blank interval. Observers searched for a single changing item among a varying number of nonchanging items until they eventually found the change. The critical manipulation in this experiment was that the size of the change was varied across trials. On some trials, the change was from a block number 2 to a block number 8 , and on others it was from a block 2 to a block 4 . The 2-to-4 change was relatively larger, because five segments changed, whereas in the 2-to- 8 case only two segments changed. As the number of items in the display was increased, the time taken to find the large change increased less rapidly than the time taken to find the small change. In other words, the different change magnitudes produced different slopes of response time versus number of distractors. The findings were taken to suggest that as the observers search for the change, their attention is implicitly attracted by an accumulation of the change signal, and the large change has a stronger attractive power. Note that this experiment represents the only claim for implicit detection examined here that does not adopt the dissociation paradigm (Reingold \& Merikle,
1988). Rather than directly asking about awareness at the end of a trial, Smilek et al. assume a lack of awareness until participants respond. Note that the central claim, however, is consistent with other claims of implicit detection; changes influence performance in the absence of awareness of these changes.

\section{Experiment 2: Better-Than-Chance Implicit Localization?}

To assess whether observers show better-than-chance localization in the absence of awareness of the change, we must first determine what constitutes chance performance. In the experiments conducted by Fernandez-Duque and Thornton (2000), observers were given a two-alternative forced-choice judgment task. Their claim for implicit localization was based on the finding that observers show better than $50 \%$ performance on this task even when they report not seeing the change. This estimate, however, does not account for all explicitly available information. As noted by the authors, on those trials for which observers reported not seeing the change, they still might have known that some items in the display did not change. If, in the process of trying to find the change, observers successfully determined that some items had not changed, they should never select those items. Consequently, if an eliminated item were one of the two options, observers would know that it had not changed and would select the other item, although they had not seen the change. This process of elimination would lead to greater than $50 \%$ performance even if observers had no implicit knowledge of the change location (see FernandezDuque \& Thornton, 2000, Footnote 5). In other words, explicit reports of change detection might not be an exhaustive measure of all explicit contributions to change detection. Simply because observers fail to report noticing a change does not mean that they lack any explicit information about the change. Consequently, the guessing measure might not be an exclusively implicit measure of detection (Reingold \& Merikle, 1988); explicit strategies might influence performance.

This argument can be made more concrete if we assume, for example, that on each trial, observers attend to two items in the display. We consider two items to be a reasonable estimate, because other studies involving the same sorts of stimuli and displays have shown that observers can attend to and hold approximately two to three items in memory from one display to the next (Rensink, 2000b). We assume further that (a) if either of these two items changed, observers would notice it, and (b) if they did not change, observers would know that they were unchanged. In the Fernandez-Duque and Thornton (2000) experiments, after every trial, the changed item and the diametrically opposite item were always cued. Therefore, if the attended item had changed and was subsequently cued, observers would correctly report detection $100 \%$ of the time. Alternatively, if the attended item did not change and subsequently became cued, observers would report that they did not see the change and would select the diametrically opposite item. Assuming that observers would not select an item they know did not change, their guess on these trials would always be correct, because the diametrically opposite item must have changed (see Footnote 5 of Fernandez-Duque \& Thornton, 2000, for a similar argument).

Given these assumptions, for a display with 8 items, the change would be detected and observers would report seeing it on 2 out of 8 of the trials. On the remaining 6 out of 8 the observers would report guessing. For two out of six of these guess trials, the 
attended item would be highlighted, and observers would correctly guess the other object $100 \%$ of the time. For the remaining 4 out of 6 trials, they would be correct, on average, on $50 \%$. Thus, on average, when reporting guessing, observers would be correct on $2 / 6+(.5 \times 4 / 6)$ of the trials, or $66.67 \%$. Likewise, for a 12 -item display, when observers report guessing, they should accurately locate the change on $2 / 10+(.5 \times 8 / 10)$, or $60.0 \%$, of the trials. For a 16-item display, accuracy when guessing will be reduced to $2 / 14+(.5 \times 12 / 14)$, or $57.14 \%$. Note that these results are based on the reasonable assumptions that observers can hold two items from one display to the next and that they will use this explicitly available information when responding. These predicted guessing levels closely mirror the actual results of the Fernandez-Duque and Thornton (2000) experiment (see Table 1), with no contribution from implicit change detection. ${ }^{3}$

The present experiment sought to provide empirical support for the theoretical possibility that explicit elimination contributes to guessing performance. On each trial, observers received 1, 2, 4, or 6 cycles of the original and changed display in a flicker task. On $75 \%$ of the trials one item in the display rotated in the picture plane, and on $25 \%$ of the trials the display contained no change. If observers detected the change, they reported which item had changed. Some observers were asked to guess the change location when they did not see the change (guess condition). Other observers were asked to report which items they knew did not change when they did not see the change (eliminate condition). The guess condition provided an estimate of localization performance in the absence of awareness of the change. The eliminate condition provided an estimate of what would constitute chance guessing. If accurate guessing is to provide any support for implicit localization, observers must guess the change location more frequently than would be expected on the basis of such explicit elimination strategies. By varying the number of exposures to the change, we can determine whether the number of items eliminated on each cycle predicts the levels of accurate guessing. By the assumptions described earlier, observers should eliminate more items with additional cycles. Consequently, their guessing performance when they do not see a change should improve by a corresponding amount.

\section{Method}

Participants. Forty-seven paid volunteers participated in a single 45min testing session. Data from 2 participants in the guess condition and 6 participants in the eliminate condition were removed as a result of subcriterion performance (as described subsequently), leaving a total of 19 participants in the guess condition and 20 in the eliminate condition (the

Table 1

Percentage of Accurate Guessing as a Function of the Number of Items in the Display

\begin{tabular}{lccc}
\hline & \multicolumn{3}{c}{ Number of items } \\
\cline { 2 - 4 } Source & 8 & 12 & 16 \\
\hline Fernandez-Duque and Thornton (2000) & 63 & 58 & 55 \\
Predicted from elimination of two items & 66.67 & 60.00 & 57.14 \\
\hline
\end{tabular}

Note. The findings of Fernandez-Duque and Thornton (2000) do not exceed the predicted levels of chance given the assumption that observers can eliminate two items in the display with each exposure to the change. number eliminated from each condition was not significantly different: Fischer exact test, $p=.154$ ).

Materials and procedure. In the current and all remaining experiments, displays were presented on the 15 -in. $(38-\mathrm{cm})$ monitor of an iMac computer with a resolution of $640 \times 480$ and a refresh rate of $117 \mathrm{~Hz}$. The stimuli consisted of a set of circular arrays composed of eight radial gratings evenly spaced around a central point. Assuming a viewing distance of approximately $50 \mathrm{~cm}$, the center of each grating was $7.28^{\circ}$ from the central point, and each grating subtended a visual angle of $4.55^{\circ}$. The individual gratings were identical to those used in Experiment 1 except that they comprised two cycles rather than six (see Figure 1). In the current experiment, the gratings underwent a $120^{\circ}$ phase shift when they changed. As in Experiment 1, observers viewed the displays from a comfortable, unconstrained distance.

Participants completed at least 14 practice trials followed by 256 test trials. Of the test trials, 192 contained a change, with the change occurring equally often in each of the eight locations. Observers were not told that no-change trials would be included, but if they asked, they were told so and encouraged to try to find a change on every trial.

On any given trial, observers viewed 1, 2, 4, or 6 cycles of the change (64 trials each). A cycle consisted of an original display of eight gratings for $250 \mathrm{~ms}$ followed by a $90-\mathrm{ms}$ blank screen of the same luminance as the background of the arrays and then a second display. If the trial contained additional cycles, the second display remained visible for $250 \mathrm{~ms}$ and was followed by a blank screen before a return to the first display. On all trials, the final display remained visible until the observer responded. Observers were allowed to rest after any trial, and each trial was initiated by the observer.

Observers were told that whenever they detected the change, they should press the $S$ key and then identify the changed item using the mouse. All observers were instructed to adopt a liberal criterion for detection: If they were mostly certain that they saw the change, they should respond saw. Observers in the guess condition were told that whenever they did not see the change, they should use the mouse to guess which grating had changed. Observers in the eliminate condition were told that whenever they did not see the change, they should use the mouse to identify all of the items they knew did not change. Observers in the eliminate condition were asked to adopt a conservative criterion for identifying eliminated items: They should identify an item only if they were certain that it did not change during the trial. Our instructions were intended to minimize guesses that might contain some explicit awareness and to determine the minimal number of items that could be eliminated with certainty.

Observers' data were eliminated if their false alarm rate was too high. A high false alarm rate would suggest that an observer was using too liberal a criterion for reporting detection of a change. Data from 1 observer in the guess condition (false alarm rate: $87.5 \%$ ) and 6 observers in the eliminate

\footnotetext{
${ }^{3}$ Note that two of our assumptions need not hold perfectly. Even if observers do not always detect the change when it involves one of the held items or do not successfully eliminate a held item when it did not change, performance can still be affected by this explicit information. In fact, it is unlikely that observers apply this exclusion strategy perfectly. In the original study, observers reported noticing the change on $52 \%$ of the eight-item trials (Fernandez-Duque \& Thornton, 2000, Experiment 3). At this level, an explanation based on held information would need to argue that observers held onto $50 \%$ of the items in the display. Yet, if they did so, they should have been able to apply the elimination strategy on every trial for which they did not notice the change. That is, they should have guessed with $100 \%$ accuracy when given a two-alternative forced choice. That performance was far worse than $100 \%$ suggests that observers were not applying the elimination strategy perfectly and that some information might have been lost if observers did not notice the change. The key to our argument, however, is that such explicit information is potentially available and that observers can use it, even if they do not use it perfectly.
} 
condition (mean false alarm rate: $55.47 \%$ ) were eliminated because their false alarm rates were more than three standard deviations away from the mean of the other observers in their condition. The data from an additional observer in the guess condition were eliminated because when he reported seeing the change, he correctly located it only $12 \%$ of the time.

\section{Results and Discussion}

Overall, observers in the guess condition reported seeing the change on only $24.51 \%(S D=7.78 \%)$ of the change trials, and on those trials they correctly identified the changed item $87.73 \%$ $(S D=5.36 \%)$ of the time. Observers rarely responded saw on the no-change catch trials $(M=4.52 \%, S D=5.23 \%)$. For the $1-, 2-$, 4-, and 6-cycle conditions, observers reported seeing the change on $5.92 \%, 14.64 \%, 32.93 \%$, and $47.53 \%$ of the trials, respectively, $F(3,54)=201.10, p<.001$. Correspondingly, with more exposure to the change, observers were more likely to correctly locate the change when reporting saw $(60.66 \%, 84.05 \%, 92.28 \%$, and $87.08 \%$ for the 1-, 2-, 4-, and 6-cycle trials, respectively), $F(3$, 54) $=151.94, p<.001 .^{4}$

Overall performance by observers in the eliminate condition was comparable. They reported seeing the change on $29.51 \%$ $(S D=7.44 \%)$ of the trials, and when they did report saw, they identified the changed object accurately $(M=92.01 \%, S D=$ $7.84 \%)$. False alarm rates were also low $(M=6.02 \%, S D=$ $6.42 \%$ ). As in the guess condition, observers reported seeing the change more frequently with increasing numbers of cycles $(9.22 \%$, $17.97 \%, 36.88 \%$, and $53.98 \%$ for the 1-, 2-, 4-, and 6-cycle trials, respectively), $F(3,57)=206.34, p<.001$. They were also more likely to correctly localize the change when responding saw with increasing exposure $(65.98 \%, 88.60 \%, 93.00 \%$, and $97.51 \%$ for the 1-, 2-, 4-, and 6-cycle trials, respectively), $F(3,57)=317.36$, $p<.001$.

More important, when observers in the guess condition responded guess, they correctly selected the changed item more frequently after viewing more cycles, $F(3,54)=6.62, p=.001$. After viewing 1, 2, 4, and 6 cycles, observers accurately guessed on $16.37 \%, 14.99 \%, 20.70 \%$, and $26.25 \%$ of the trials, respectively. According to the logic of the original claims for implicit localization, chance should be 1 out of 8 , or $12.5 \%$. Thus, the rate of accurate guessing was better than would be expected by an arbitrary guess, and that rate increased with additional exposure to the change.

To determine whether explicit strategies account for the betterthan-chance guessing as well as the improvements in guessing, we can determine the level of accurate guessing that would be predicted by an explicit elimination strategy. If observers never guess items that were eliminated, then assuming observers can eliminate some items on each cycle, guessing rates should be consistently greater than $12.5 \%$ and should increase with additional exposure to the change. The predicted level of guessing accuracy should be $1 /(N-$ Number Eliminated), where $N$ is the number of items in the display. Therefore, with eight items in the display, if no items were eliminated, chance would be 1 out of 8 [1/(8 - 0)], or $12.5 \%$; if one item were eliminated, chance would be 1 out of 7 [1/(8 - 1)], or $14.29 \%$; if two items were eliminated, chance would be 1 out of $6[1 /(8-2)]$, or $16.67 \%$; and so on.

Consistent with the explicit elimination account, participants in the eliminate condition were able to rule out more items with additional exposure, $F(3,54)=145.30, p<.001$. For 1-, 2-, 4-, and 6-cycle trials, they were able to eliminate an average of 1.03, $1.93,3.58$, and 4.11 items, respectively. ${ }^{5}$ Therefore, the elimination strategy would predict accurate guessing rates of $14.45 \%$, $16.80 \%, 23.42 \%$, and $27.48 \%$ for 1-, 2-, 4-, and 6-cycle trials, respectively. As can be seen in Figure 2, the predictions of the eliminate condition corresponded to the levels of accurate guessing nearly perfectly. There was no main effect of condition (guess vs. eliminate), $F(1,37)=0.56, p=.459$, nor was there an interaction between condition and amount of exposure, $F(3,111)=0.86, p=$ .465 .

In summary, with more exposure to the change, observers were more likely to detect the change. Also, with more exposure but without explicit detection, the guess group was more accurate, and the eliminate group ruled out more items. It is important to note that the rate of accurate guessing did not exceed the levels predicted by a strategy of explicit elimination. Given the theoretical argument that elimination can explain the original result and the empirical evidence that an explicit strategy predicts accurate guessing rates in the absence of explicit change detection, existing evidence for better-than-chance guessing does not unequivocally support a claim of implicit localization. Accurate guessing is no better than would be expected on the basis of explicit strategies.

\section{Experiment 3: \\ Implicit Accumulation of Location Information}

The results of Experiment 2 suggest that implicit localization, if it exists, does not contribute to explicit localization as measured by better-than-chance guessing. Experiment 3 tested the other published claim about implicit localization. The experiment specifically tested whether or not focused, explicit comparison processes can account for differences in search slopes for large and small changes. As noted earlier, when observers search for a changing item, their detection times are less affected by the number of distractors in the display when the change is larger (Smilek et al., 2000). This finding has been interpreted as evidence for the implicit accumulation of change information before awareness. The change is implicitly localized, and information for the change accumulates, thereby guiding attention to the change location. Given that larger changes produce a greater signal, they accumulate faster, thereby guiding attention to the change location more rapidly.

Although implicit accumulation might well produce such a search slope difference, an alternative mechanism that relies on explicit, serial search and requires no implicit change detection also predicts this result. Even with focused attention, changes are

\footnotetext{
${ }^{4}$ Note that these and the following omnibus analyses do not necessarily signify an increase in the number of responses with an increase in exposure to the change. Rather, they simply indicate the existence of a difference among the means. To verify that these differences reflected increasing responses, we calculated, for each observer, the correlation between his or her responses and the actual number of exposures (1, 2, 4, and 6). For all of the omnibus analyses reported in the current section, the mean correlation was significant and had an average value of .936 .

${ }^{5}$ Given the differences between our radial grating stimuli and Fernandez-Duque and Thornton's (2000) oriented bar stimuli, it is not surprising that fewer items could be held across displays. Also note that the number of items eliminated, or held, appeared to asymptote at four (Rensink, 2000b).
} 


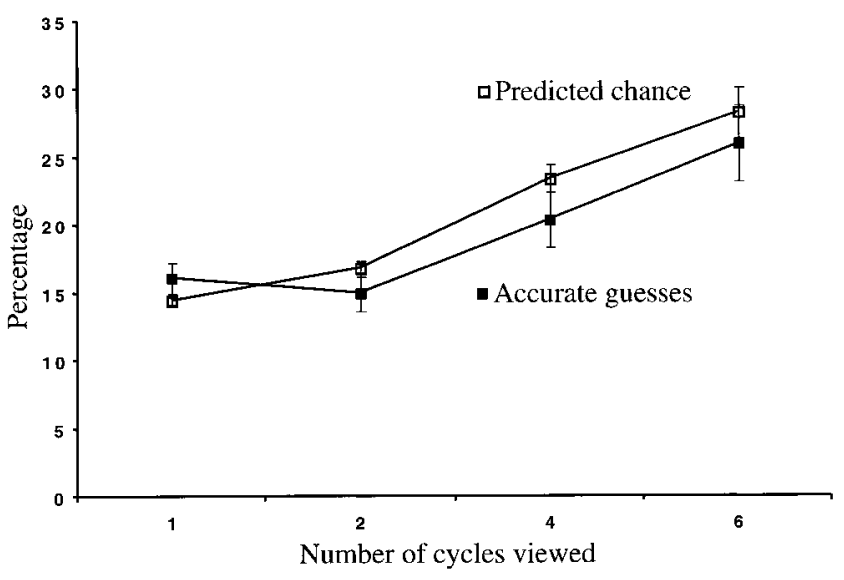

Figure 2. Mean percentages of accurate guesses from the guess condition of Experiment 2, along with the predicted levels of chance calculated from performance in the eliminate condition. Performance in both groups increased with additional exposure to the change. Error bars represent standard errors of the means.

not always detected (Levin \& Simons, 1997; Simons \& Levin, 1998; Williams \& Simons, 2000); with a single changing object in the display, detection is better when more parts of the object are changed, and it is not perfect even when several parts are changed (Williams \& Simons, 2000). Given the effect of change magnitude on detection of changes to attended objects, explicit, serial search will produce a search slope difference even with no implicit detection. If small changes are more difficult to detect with focused attention, then a brief scan of an item with focused attention will be more likely to miss a small change than a large change. Whenever a change is missed, a serial search would need to return to that item at some later point, thereby requiring more time overall. Consequently, searches for smaller changes will have steeper search slopes. Note that this effect should occur even if observers have no knowledge that the changes are of different magnitudes across trials (see Mitroff \& Simons, in press, for a more formal analysis).

We tested this alternative, nonimplicit hypothesis empirically by creating a case in which accumulation is impossible. If search slopes are still different for small and large changes, then an explicit account would be more parsimonious. The three parts of this experiment compared three different conditions. Experiment $3 \mathrm{~A}$ was a direct replication of the original result reported by Smilek et al. (2000). Experiment 3B modified the original design of the experiment. Rather than continuously flickering displays being presented, on each trial the presentation was stopped after each cycle of change (original, blank, and changed). After each cycle, observers indicated whether or not they detected the change. If they detected the change, the trial ended. If they did not see the change, they viewed additional cycles, one at a time, until they did detect the change. Assuming that the posited implicit accumulation mechanism is not overly sensitive to these temporal disruptions required for responses, this modified task should still produce the slope difference (note that the explicit account predicts the slope difference here as well). Experiment $3 \mathrm{C}$ directly tested the implicit accumulation hypothesis by removing any possibility of accumulation across repeated exposures to the change. The method was identical to that of Experiment $3 \mathrm{~B}$ except that, on each additional cycle, the location of every item in the display was randomized. Consequently, if the search slope difference results from implicitly accumulating location information, the slope difference should disappear entirely in Experiment 3C, because the location varied with each exposure to the change. In contrast, if a slope difference persists, then it must be due to the differential detectability of small and large changes, presumably with focused attention.

\section{Experiment 3A: Replication of Smilek et al. (2000)}

\section{Method}

Participants. Twenty individuals were recruited through posted signups and were paid $\$ 7$ for their participation. To be included in analyses, participants were required to meet a criterion of $80 \%$ correct localization when they reported seeing a change. All participants met this criterion in the present experiment, in part because they could always view an additional cycle to verify that they had seen the change.

Materials and procedure. As in the original study (Smilek et al., $2000)$, all of the objects in a display were randomly assigned to positions in an imaginary $6 \times 6$ grid $(8.1 \mathrm{~cm}$ vertical $\times 6.99 \mathrm{~cm}$ horizontal). From an approximate viewing distance of $50 \mathrm{~cm}$, the entire grid subtended $9.28^{\circ}$ vertical $\times 8.01^{\circ}$ horizontal. Each object within the grid subtended an approximate visual angle of $1.09^{\circ}$ vertical and $0.73^{\circ}$ horizontal. Each display contained 1 changing item and equal numbers of each type of distractor item (see Figure 1). For example, a 7-item display had the changing item along with two $2 \mathrm{~s}$, two $4 \mathrm{~s}$, and two 8 s, and a 13-item display had the changing item and four $2 \mathrm{~s}$, four $4 \mathrm{~s}$, and four $8 \mathrm{~s}$. All items were light gray $\left(53 \mathrm{~cd} / \mathrm{m}^{2}\right)$ and were presented against a black $\left(0.5 \mathrm{~cd} / \mathrm{m}^{2}\right)$ background. As noted earlier, the magnitude of a change was either large (changing a block 2 to a block 4 , or vice versa) or small (changing a block 2 to a block 8 , or vice versa).

Observers pressed a key to begin each trial. An initial display was presented for $200 \mathrm{~ms}$, followed by a blank screen of the same color as the background for $80 \mathrm{~ms}$, the modified display for $200 \mathrm{~ms}$, and then another 80 -ms blank screen. This sequence repeated continuously until the observer pressed a key to indicate detection. After the sequence stopped, observers used the mouse to click on the changing item (if the alternation was stopped during a blank interval, the subsequent display was presented for this mouse click).

Observers viewed as many practice trials as needed and then completed 300 randomly ordered test trials, 60 of each of five set sizes $(4,7,10,13$, and 16). Of the 60 trials for each set size, 30 contained a large change (15 trials from a block 2 to a block 4 and 15 from a 4 to a 2), and 30 contained a small change (15 trials from a 2 to an 8 and 15 from an 8 to a 2). Observers were prompted to rest after every 50 trials and were instructed to take breaks as needed.

\section{Results and Discussion}

Observers made relatively few localization errors in this experiment $(M=2.05 \%, S D=1.10 \%)$. Error rates were somewhat greater when the change was small $(M=2.75 \%)$ than when it was large $(M=1.33 \%), t(19)=3.63, p=.001$, but it is important to note that there was no significant interaction between magnitude of the change and set size in the case of number of localization errors, $F(4,76)=1.97, p=.109$. Consequently, differences in the search slopes for small and large changes cannot be attributed to differences in error rates. Trials with localization errors were eliminated from analyses of response latencies. As in the original study, response time outliers were eliminated through a recursive procedure: For each observer, the slowest response time in each set-size category was eliminated. If the removed time was more than four standard deviations from the mean for a given set size calculated 
without that item, the item was permanently removed. This procedure was repeated with the remaining items until a removed item was fewer than four standard deviations from the mean for that set size. On average, $0.87 \%$ of each participant's trials were eliminated $(S D=0.77 \%)$. More large-change trials $(M=0.94 \%)$ than small-change trials $(M=0.81 \%)$ were eliminated, but the difference was not significant, $t(19)=0.77, p=.449$.

The pattern of results for response times was comparable to that of Smilek et al.'s (2000) experiments (see Figure 3A and Figure 3B). Larger changes were found faster $(M=1,911 \mathrm{~ms})$ than smaller changes $(M=2,256 \mathrm{~ms}), t(19)=7.76, p<.001$. More important, as the number of items in the displays increased, the relative difference between large and small changes also increased, resulting in a shallower search slope for the large changes (124.50 $\mathrm{ms}$ per item) than for the small changes (162.71 ms per item),

A

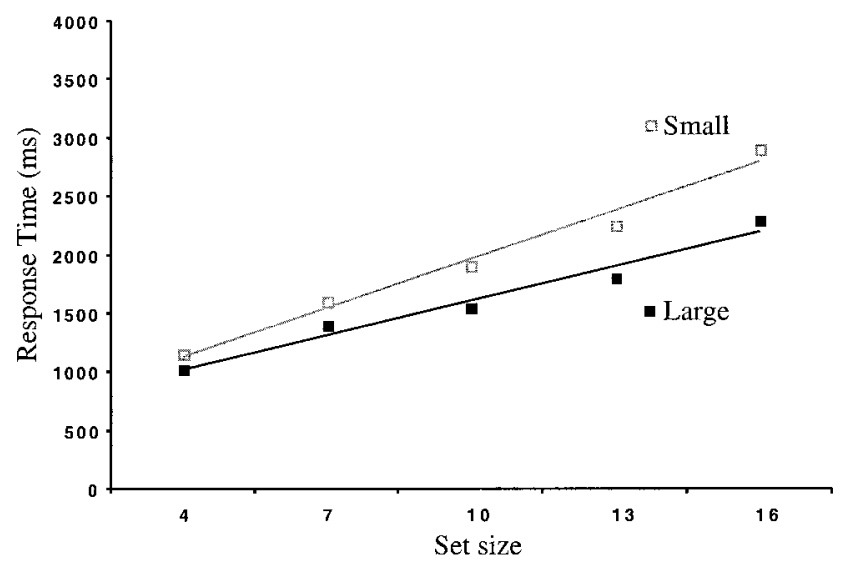

$\mathrm{C}$

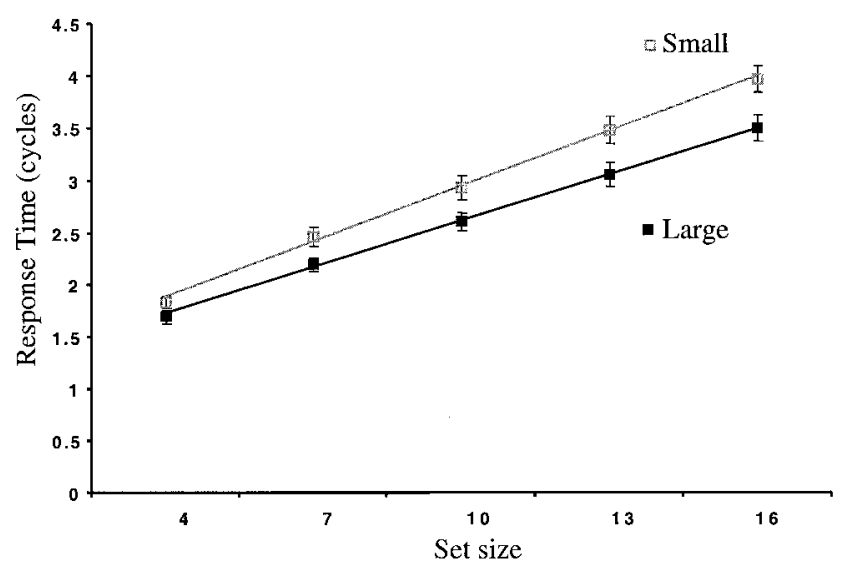

$t(19)=4.87, p<.001$. Of the 20 observers, 19 exhibited a shallower slope for large than for small items. Thus, Experiment $3 \mathrm{~A}$ replicated the original result despite several small changes to the procedure (e.g., using a mouse for localization rather than typing grid numbers).

\section{Experiment 3B: Modified Version of Replication}

This experiment introduced a slight modification of the method used in Experiment 3A. The goal of this change was to verify that the slope difference between large and small changes persists even when observers are asked to report whether or not they detected the change after each cycle. If so, we could then proceed to examine the implicit accumulation model directly in Experiment $3 \mathrm{C}$.

\section{B}

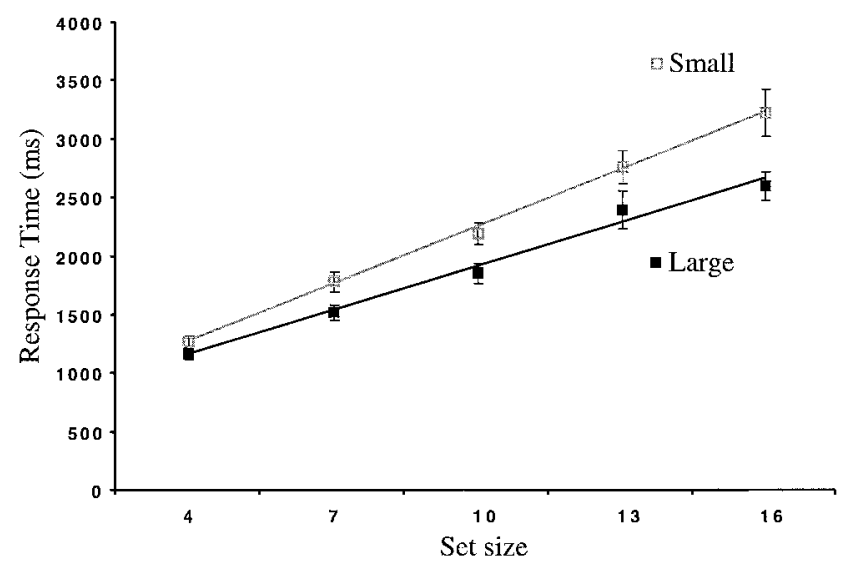

$\mathrm{D}$

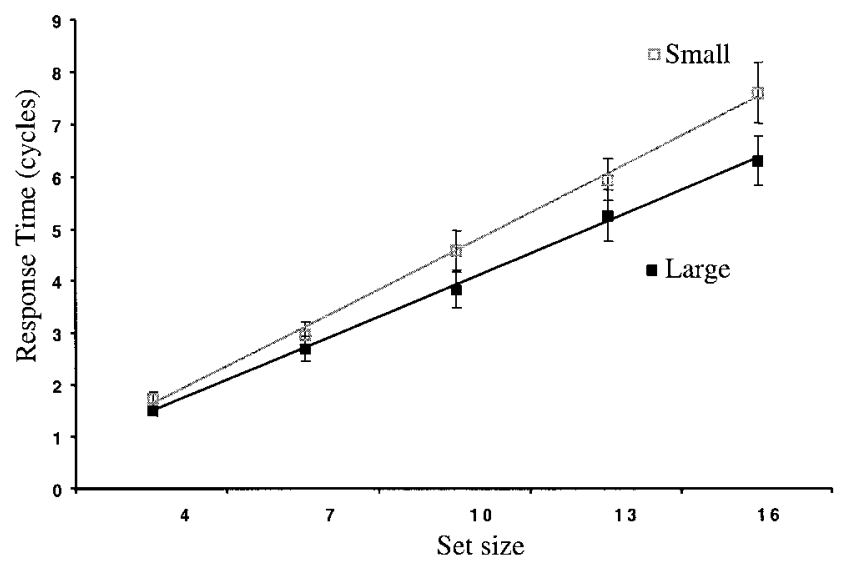

Figure 3. Data from Experiment 3. A: Results of the study by Smilek et al. (2000, Experiment 2). Adapted from "Does Unattended Information Facilitate Change Detection?" by D. Smilek, J. D. Eastwood, and P. M. Merikle, 2000, Journal of Experimental Psychology: Human Perception and Performance, 26, p. 485. Copyright 2000 by the American Psychological Association. Adapted with permission of the author. B, C, and D: Results of Experiments 3A, 3B, and 3C, respectively. Error bars represent standard errors of the means for each set size. Note that response time is shown as number of cycles in $\mathrm{C}$ and $\mathrm{D}$ and that these values are on different scales from each other and from the other panels. Given the added difficulty of the task, the slopes for Experiment $3 \mathrm{C}$ (D) are steeper than those for Experiment 3B (C). 


\section{Method}

Participants. Twenty-one individuals were recruited through posted sign-ups and were paid $\$ 7$ for their participation. As in Experiment 3A, inclusion in the analyses required that participants meet a criterion of $80 \%$ correct localization when they reported seeing a change. Only 1 participant failed to meet this criterion (as in Experiment 3A, observers could always view an additional cycle to verify that they had seen the change, and consequently error rates were relatively low). The final analyses included data from 20 participants.

Materials and procedure. The displays and procedure of this experiment were identical in all respects to those of Experiment 3A, with one exception. On each trial, observers viewed a single cycle of the change (original, blank, and modified). The modified display remained visible until the observer responded. If observers detected the change, they were asked to press the $S$ key and then to localize the changed item. If they did not detect the change, they were asked to press the space bar to view another cycle. An 80-ms blank screen was presented, followed immediately by another cycle of the change. This procedure was repeated until observers pressed the $S$ key or until they had viewed 30 cycles, whichever came first.

\section{Results and Discussion}

Similar to Experiment 3A, localization errors occurred on an average of only $2.02 \%$ of the trials $(S D=1.72 \%$ ). Although participants made slightly more errors on small-change trials $(2.09 \%)$ than on large-change trials $(1.91 \%)$, the difference was not significant, $t(19)=0.68, p=.418$. Again, there was no significant interaction between magnitude of the change and set size in the case of number of localization errors, $F(4,76)=0.43$, $p=.787$.

As a result of the discrete presentations of each cycle, in both the current experiment and Experiment 3C, response time was measured as the number of cycles needed for detection rather than in milliseconds. Outlier response times were removed in the same manner as in Experiment 3A. On average, $0.14 \%$ of each observer's response times were eliminated $(S D=0.21 \%)$. Slightly more response times were eliminated for small changes $(0.16 \%)$ than for large changes $(0.11 \%)$, but the difference was not significant, $t(19)=1.17, p=.258$.

The pattern of results in Experiment 3B was consistent with that of Experiment 3A. On average, large changes ( $M=2.62$ cycles) were found faster than small changes ( $M=2.94$ cycles), $t(19)=$ $6.85, p<.001$. More important, the relative difference between search times for large and small changes increased with more items in the display, such that the slope for large changes $(0.149$ cycles per item) was shallower than that for small changes $(0.176$ cycles per item), $t(19)=3.48, p=.003$. Of the 20 observers, 15 exhibited a shallower search slope for large changes than for small changes. Thus, the pattern of results was comparable to that of Experiment $3 \mathrm{~A}$, even with the added delay introduced by presenting the changes one cycle at a time.

\section{Experiment 3C: Testing the Accumulation Model}

If the difference in slopes for large and small changes found in the original work and in Experiments $3 \mathrm{~A}$ and $3 \mathrm{~B}$ results entirely from the implicit accumulation of change location information as observers repeatedly view a change, then performance should depend on the constancy of the position of the changing item. That is, implicit accumulation should be tied to the location of the changing item; a slope difference should occur only when the change is in the same location across repeated exposures. Here we randomized the position of the change on each cycle, thereby eliminating the possibility of implicit accumulation with repeated exposures. If the slope difference persists despite the randomized presentation, it cannot be attributed to an implicit accumulation of information operating to draw attention to the location of the change.

\section{Method}

Participants. Thirty-four individuals were recruited through posted sign-ups and were paid $\$ 7$ for their participation. As in Experiments $3 \mathrm{~A}$ and $3 \mathrm{~B}$, inclusion in the analyses required that participants meet a criterion of $80 \%$ correct localization when they reported seeing a change. In this experiment, observers could not verify whether or not they had seen the change, because each cycle of change randomized the change location. Consequently, error rates were higher in this experiment, and 13 participants failed to meet the accuracy criterion. One additional participant failed to complete the entire experiment. Data from these participants were eliminated, so the final analyses included data from 20 participants.

Materials and procedure. The displays and procedure of this experiment were identical in all respects to those of Experiment 3B, with one exception. On each cycle, all of the items in the display were randomly reassigned to locations in the imaginary $6 \times 6$ grid (see Horowitz \& Wolfe, 1998 , for a similar method used to study the role of memory in visual search). Furthermore, although the change magnitude (large or small) remained constant during a trial, the direction of the change (e.g., 2 to 4 or 4 to 2) was determined randomly for each cycle. Because the locations of all items in the display, as well as the change sequence, were randomized, change information could not accumulate across repeated cycles.

\section{Results}

The general pattern of results was identical to that of Experiment $3 \mathrm{~B}$ in terms of both errors and response times. Accurate localization in this experiment was more difficult, because observers could not verify the change location by viewing an additional cycle. Consequently, as mentioned, data from 13 observers were eliminated because these individuals exceeded our error rate criterion of $20 \%$. Among the remaining observers, error rates were somewhat higher overall $(M=10.83 \%, S D=6.24 \%)$ than in earlier experiments, but the pattern was comparable to those of Experiments 3A and 3B (note that absolute levels of error rates are not terribly meaningful given the number of observers whose data were excluded). These observers made more errors when the change was small $(M=11.59 \%)$ than when it was large $(M=$ $10.1 \%), t(19)=2.16, p=.043$; again, however, there was not a significant interaction between magnitude of change and set size, $F(4,76)=0.41, p=.803$.

Response time outliers were eliminated in the same manner as in Experiments 3A and 3B. On average, $0.41 \%$ of each participant's trials were eliminated $(S D=0.37 \%)$. Again, more trials were eliminated for large changes $(M=1.23 \%)$ than for small changes $(M=0.43 \%), t(19)=5.43, p<.001$. Although this difference was significant, the number of trials eliminated was minimal. In addition, if observers did not find the change after 30 cycles, the trial ended and they moved on to the next trial. No such aborted trials occurred in Experiment 3B, but an average of 3.1 aborted trials per observer occurred here. This was expected given the increased difficulty of the current experiment. No data from these trials were included in the analyses. 
As in Experiment 3B, analysis of slope differences was based on number of cycles needed to detect the change rather than on time in milliseconds. Although this measure might be somewhat less sensitive than response time, it was sufficient to replicate the critical pattern of slope differences in Experiment 3A. Although the positions of the items were randomized on each cycle, the pattern was nearly identical to that of Experiment 3B. On average, observers needed more cycles to detect small changes $(M=4.51)$ than to detect large changes $(M=3.89), t(19)=3.80, p=.001$. As the number of items in the display increased, observers required relatively more cycles to detect small changes $(0.490$ cycles per item) than large changes (0.404 cycles per item), $t(19)=2.63, p=$ .016. Of the 20 observers, 16 exhibited a shallower search slope for large changes than for small changes. Thus, even with the positions of all of the items randomized on each cycle, search slopes for large changes were still shallower than those for small changes (see Figure 3 for a comparison of the results of Experiments $3 \mathrm{~A}-3 \mathrm{C})$.

\section{Discussion}

This experiment replicated the original slope difference between large and small changes, but with no possibility for the accumulation of information across cycles. Consequently, the slope difference must be explained in terms of the differential detectability of large and small changes within a single cycle. Given that such a slope difference can arise without implicit localization, we can conclude that earlier claims of implicit localization were premature. The results of our studies, as well as those of the earlier studies (Smilek et al., 2000), are entirely consistent with a model in which changes are detected solely through an explicit comparison mechanism, with no contribution from implicit detection or accumulation. As in studies of change detection for attended objects (Williams \& Simons, 2000), larger changes are easier to detect.

Although Experiment 3C disproves the need for implicit accumulation across cycles to produce a slope difference, it does not eliminate the possibility that implicit detection guides explicit localization within a single cycle. A change signal may well exist on any given cycle, and it may be larger for larger changes. This signal, in principle, could be implicitly localized before it is explicitly detected. However, this form of implicit processing is quite different from the implicit accumulation posited on the basis of the slope differences; it does not require any accumulation of either change location or identity information over time. Although this is a logical possibility, none of the studies to date provide any compelling evidence supporting the existence of such an implicit detection mechanism. In fact, the existing evidence suggests that performance is no different than would be expected from an explicit comparison process alone.

\section{CONCLUSIONS: IMPLICIT LOCALIZATION}

The two strongest claims for implicit localization are based on evidence that, when examined more closely, can be explained without any need to posit implicit change detection. Observers can guess the change location more than $50 \%$ of the time when given a two-alternative choice, but this pattern can be explained by explicit elimination strategies. Furthermore, such elimination strategies predict the increase in guessing accuracy when observers are given more exposure to the change. In a similar manner, claims that change information implicitly accumulates and draws attention with additional exposure to the change are based on evidence for a search slope difference between large and small changes. Yet, these results can be explained by the differential explicit detectability of large and small changes within focused attention. When we eliminated any possibility of accumulation across repeated presentations of a change, observers still showed a difference in search slopes for large and small changes. Consequently, our studies support the idea that these results can be explained by explicit factors without appeal to implicit change detection. These results are in agreement with our earlier finding (Mitroff \& Simons, in press) that change localization does not improve with repeated exposures, provided observers have not yet reported noticing the change.

Taken together, the first three experiments provide evidence that all previous findings of implicit change registration and implicit change localization can also be explained by explicit processing. The present findings demonstrate that these approaches to measuring implicit change detection have not exhaustively eliminated explicit contributions to performance. In the absence of unequivocal evidence for implicit registration and localization, the only remaining class of implicit claims involves implicit identification. The next section addresses this possibility.

\section{EXPERIMENT 4: IMPLICIT IDENTIFICATION}

Although changes do not appear to be localized or registered implicitly, the nature of the change itself might be implicitly detected; without any explicit awareness of the change, the nature or identity of the change could affect the observer's behavior. Thornton and Fernandez-Duque (2000) used a modified version of the change localization task discussed in Experiment 2 to provide evidence for such an implicit effect of the identity of a change. Observers viewed an array of rectangles followed by a blank screen and then by another array of rectangles. On two thirds of the trials, one rectangle changed orientation from vertical to horizontal, or vice versa. After the change, one rectangle was cued briefly, and observers were asked to report the orientation of the cued rectangle. Then observers reported whether or not they detected the change during the trial. The critical question in this experiment was whether or not the identity of the changed item (horizontal or vertical after the change) influenced performance on the orientation judgment task. The prediction of the implicit identification hypothesis is that observers should be faster to judge the orientation of a cued line when the orientation of the changed item was the same as that of the cued item, and this effect should occur regardless of whether or not observers detected the change. The identity of the changed item presumably is registered automatically even if it is not explicitly detected, and then, if it is inconsistent with the cued item, it should impair the orientation judgment task.

Consistent with this hypothesis, observers made more errors on the orientation judgment task if the cued item had an orientation different from that of the changed item. For example, if the changed item was horizontal on the second display and the cued item was vertical, observers more often mistakenly responded horizontal. Even when observers did not consciously detect the change, they made more errors when the identity of the changed item was incongruent with that of the cued item. 
Although this congruence effect suggests that the identity of the changed item was processed even in the absence of change detection, the results might well have been due to an aspect of the design that allows explicit contamination. In these experiments, when there was a change, the cued item was either the changed item (50\% of the trials) or the item diametrically opposite from the changed item in the circular array (50\% of the trials). Given that part of the observers' task was to report whether they had detected the change, this spatial relationship might well have mediated the congruence effect. Observers could rapidly learn this association and periodically attend to the item opposite the cued item, thereby producing a systematic difference in error rates.

If the higher error rate for incongruent trials (the cued item and the changed item are of different orientations) reflects implicit change detection, then the consistent spatial relationship between the cued item and the changed item should be unnecessary to produce the effect. Therefore, if this spatial contingency were severed, the implicit identification hypothesis should continue to predict a congruence effect. Alternatively, if the congruence effect results from registration of the spatial relationship between the changed item and the cue, then severing this predictive link should also eliminate the congruence effect.

Experiment 4A precisely replicated the original experiment of Thornton and Fernandez-Duque (2000). Experiment 4B then modified this original design by severing the spatial contingency between the cued item and the changed item. If the congruence effect results from implicit identification, it should still be found in Experiment 4B. Alternatively, if the effect results from the presence of a spatial contingency, then it should be eliminated in Experiment $4 \mathrm{~B}$.

\section{Experiment 4A: Replication of the Congruence Effect}

This experiment was designed to replicate the congruence effect that serves as the basis for claims of implicit identification (Thornton \& Fernandez-Duque, 2000, Experiment 2).

\section{Method}

Participants. Twenty individuals were recruited for this experiment and paid $\$ 7$ for their participation. As in the original experiment of Thornton and Fernandez-Duque (2000), data from any observer whose overall accuracy was more than two standard deviations from the mean were eliminated. No observers were eliminated from either Experiment 4A or Experiment 4B.

Materials and procedure. As in earlier experiments, viewing distance was unconstrained but averaged approximately $50 \mathrm{~cm}$. The displays used in this experiment consisted of arrays of eight rectangles evenly spaced in a circle of radius $7.78 \mathrm{~cm}\left(8.92^{\circ}\right)$ around a central fixation point. The rectangles each subtended $2.82^{\circ} \times 1.82^{\circ}(2.46 \mathrm{~cm} \times 1.59 \mathrm{~cm})$. The rectangles were black $\left(0.5 \mathrm{~cd} / \mathrm{m}^{2}\right)$, and the background was light gray (40 $\left.\mathrm{cd} / \mathrm{m}^{2}\right)$. The initial display consisted of four horizontal and four vertical rectangles, and the second display either was identical to the first (no change) or involved one rectangle being rotated by $90^{\circ}$ (change).

In each trial, a fixation point appeared in the center of the display, and then observers pressed a key to begin. The first array of rectangles appeared for $250 \mathrm{~ms}$, after which the rectangles disappeared, leaving only the fixation point for $250 \mathrm{~ms}$. After this blank interval, the second array appeared. Then, after $250 \mathrm{~ms}$, one item in the second array changed color from black to light gray, and then, after an additional $20 \mathrm{~ms}$, the entire array disappeared (see Figure 1). Given that the luminance change was visible for only $20 \mathrm{~ms}$ followed by the transient caused by the disappearance of the other items, the color change appeared as a brief flash and was somewhat difficult to detect. Immediately after the second display had been removed, observers were asked to report as quickly as possible whether the flashed item was horizontal or vertical by pressing one of two keys on a keyboard. They were instructed to guess the orientation if they had not seen the cue. After this orientation response, observers reported whether or not they had detected a change from the first to the second display by pressing one of two keys. The instructions stressed the primary importance of the speeded orientation decision and noted that the same-different response was not speeded and was of secondary importance.

Following Thornton and Fernandez-Duque (2000), each observer participated in three practice sessions of approximately 20 trials each (observers were allowed to continue each session until comfortable). The sessions gradually decreased the amount of time that the cued item was visible: 200 $\mathrm{ms}$ in the first session, $40 \mathrm{~ms}$ in the second session, and, finally, $20 \mathrm{~ms}$ in the third session (the same as in the test trials). The first session allowed participants to become familiar with the task, because the luminance cue was easily detected. After the practice sessions, participants completed five blocks of 128 randomly ordered test trials. Observers were given breaks between blocks and were instructed to rest during a block if needed.

Of the 640 trials, 128 were no-change trials on which the first and second displays were identical. On these trials, the luminance cue appeared equally often (16 times) at each of the eight spatial locations. In a similar manner, on the 512 change trials, the cued item appeared equally often in all eight locations (64 times each). On half of the change trials, the cued item was also the changed item (valid trials); on the other half, the cued item was diametrically opposite the changed item (invalid trials). Thus, in the absence of change detection, observers could not predict the cue's location. However, the appearance of the cue did reduce the number of possible locations for the changed item to two. On half of the invalid trials the cued item was the same orientation as the changed item in the second display (congruent cue), and on the other half the cued item was a different orientation from the changed item (incongruent cue).

\section{Results}

The primary analysis in the original study involved error rates. However, for completeness, we describe response times as well. Observers were faster to respond on invalid trials than on valid trials, regardless of whether or not they were aware of the change (see Table 2). This effect was confirmed by a 2 (validity) $\times 2$ (awareness) analysis of variance (ANOVA) that produced an almost significant main effect of validity, $F(1,19)=3.31, p=.085$, a nonsignificant main effect of awareness, $F(1,19)=1.04, p=$ .322 , and a nonsignificant interaction, $F(1,19)=0.04, p=.836$. When observers noticed the change, they were significantly faster on valid trials than on invalid trials, $t(19)=2.27, p=.035$. Observers were no faster to respond on congruent trials than they were on incongruent trials, regardless of whether or not they were aware of the change. This pattern was confirmed by a 2 (congruence) $\times 2$ (awareness) ANOVA that produced no main effect of congruence, $F(1,19)=2.55, p=.127$, or awareness, $F(1,19)=$ $0.01, p=.931$, and no significant interaction, $F(1,19)=0.22, p=$ .648 .

Given the high error rates in this experiment, analyses of response times are not a particularly useful measure. Overall, observers successfully detected $55.56 \%$ of the changes and incorrectly reported different when no change occurred on $16.68 \%$ of the no-change trials. This false alarm rate was comparable to that of the original study (Thornton \& Fernandez-Duque, 2000). The high false alarm rate does not appear to signify a lack of sensitivity, because overall observers were sensitive to changes (mean $A^{\prime}$ $=.80$ ) and were biased to respond same (mean $B^{\prime \prime}=.28$ ). Some 
Table 2

Mean Response Times (in Milliseconds) and Percentages of Error for Each Condition in Experiment $4 A$ as a Function of Awareness of the Change

\begin{tabular}{|c|c|c|c|c|}
\hline \multirow[b]{2}{*}{ Condition } & \multicolumn{2}{|c|}{ Response time (ms) } & \multicolumn{2}{|c|}{ Error rate $(\%)$} \\
\hline & Unaware & Aware & Unaware & Aware \\
\hline No change & $983(245)$ & $1,012(308)$ & $30.5(15)$ & $39.7(22)$ \\
\hline Valid & $988(270)$ & $1,001(249)$ & $24.2(16)$ & $16.3(9)$ \\
\hline Invalid congruent & $941(193)$ & 946 (199) & $26.0(16)$ & $19.3(10)$ \\
\hline Invalid incongruent & $975(205)$ & $968(214)$ & $39.9(20)$ & $53.3(10)$ \\
\hline
\end{tabular}

Note. Standard deviations appear in parentheses.

errors might also have occurred when observers confused the transient produced by the luminance cue for the change itself.

Awareness and cue validity. This analysis compared performance on change trials for which the cued item was the changed item to performance on trials in which the cued item was not the changed item but was still congruent with the changed item (incongruent trials are not considered here). In general, observers were more accurate on valid than on invalid trials, $F(1,19)=5.00$, $p=.038$, and they were more accurate when they noticed the change than when they did not notice the change, $F(1,19)=5.45$, $p=.031$; however, the validity effect did not interact with awareness, $F(1,19)=0.24, p=.626$. Furthermore, when observers were unaware of the change, they were equally accurate on valid and invalid trials, $t(19)=0.89, p=.383$.

Awareness and congruence. This analysis compared performance on change trials for which the cue was congruent or incongruent with the changed item (excluding all valid trials). The central analysis involved the effect of the congruence of the changed item and the cued item with and without awareness of the change. In general, observers made more errors on incongruent trials than on congruent trials, $F(1,19)=34.23, p<.001$. In addition, as in the previous analysis, they made fewer errors when they were aware of the change than when they were unaware of the change, $F(1,19)=4.42, p=.049$. The Congruence $\times$ Awareness interaction was also significant, $F(1,19)=16.53, p<.001$; even when observers were unaware of the change, however, they still made fewer errors on congruent trials than on incongruent trials, $t(19)=4.58, p<.001$. That is, even when they were unaware of the change, the congruence of the identity of the changed item to that of the cued item affected their performance.

\section{Discussion}

This experiment replicated the primary finding used to support a claim of implicit identification (Thornton \& Fernandez-Duque, 2000): Observers made more errors when the change was incongruent with the cued item than when it was congruent, even in the absence of awareness (see Figure 4). However, as noted earlier, the link between the spatial position of the cue and the spatial position of the change made the change location predictable from the cued location. This association might well account for the interference produced by incongruent changed items. Experiment 4B tested this nonimplicit explanation for the congruence effect.

\section{Experiment $4 B$ : \\ Testing the Implicit Identification Hypothesis}

According to the implicit identification hypothesis, a change is implicitly detected, and the identity of the changed item is implicitly processed. This processing then interferes with a decision about the orientation of the cued item. If the change is detected implicitly, the congruence effect should be unaffected by any spatial relationship between the changed item and the cued item. On the other hand, if the congruence effect occurs because observers learn the spatial contingency between the cued item and the changed item, then the congruence effect should be eliminated by randomizing the position of the changed item with respect to the cued item. According to this view, awareness of the contingency mediates the congruence effect. Consequently, when the contingency is eliminated, observers should show no congruence effect regardless of whether or not they notice the change. That is, neither the aware nor the unaware trials should show a congruence effect.

\section{Method}

Participants. Twenty individuals were recruited for this experiment and paid $\$ 7$ for their participation.

Materials and procedure. The materials and procedure of Experiment 4B were identical to those of Experiment 4A, except that the location of the changed item and that of the cued item were completely counterbalanced to eliminate any systematic spatial relationship. On change trials, the change occurred equally often at all eight locations (64 trials each). Furthermore, for each change location, the cued item appeared equally often in each of the eight locations. Thus, one eighth of the change trials (64 trials) were valid trials in which the changed item was also the cued item. In the remaining seven eighths of the change trials (448 trials), the cued item appeared equally often in each of the nonchange locations. As in Experiment $4 \mathrm{~A}$, half of the change trials were congruent and half were incongruent.

\section{Results}

As in Experiment 4A, the response times were collected and analyzed. Neither the Validity $\times$ Awareness ANOVA nor the Congruence $\times$ Awareness ANOVA revealed any significant effects. Given the difficulties in interpreting response times in the face of high error rates, the primary analyses focused on differences in errors across conditions. Overall, observers detected the change on $68.99 \%$ of the change trials and produced false alarms on $23.24 \%$ of the no-change trials. Observers were again biased to 
A

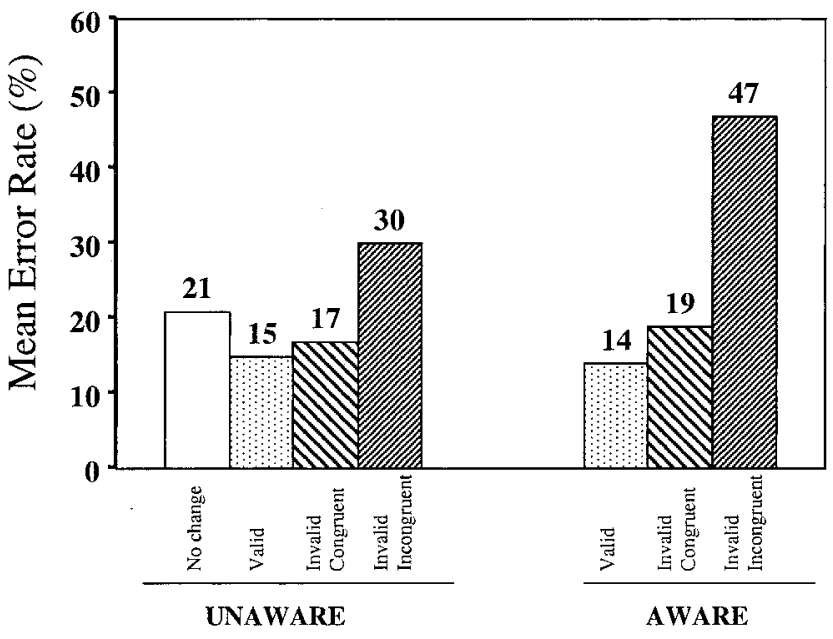

B

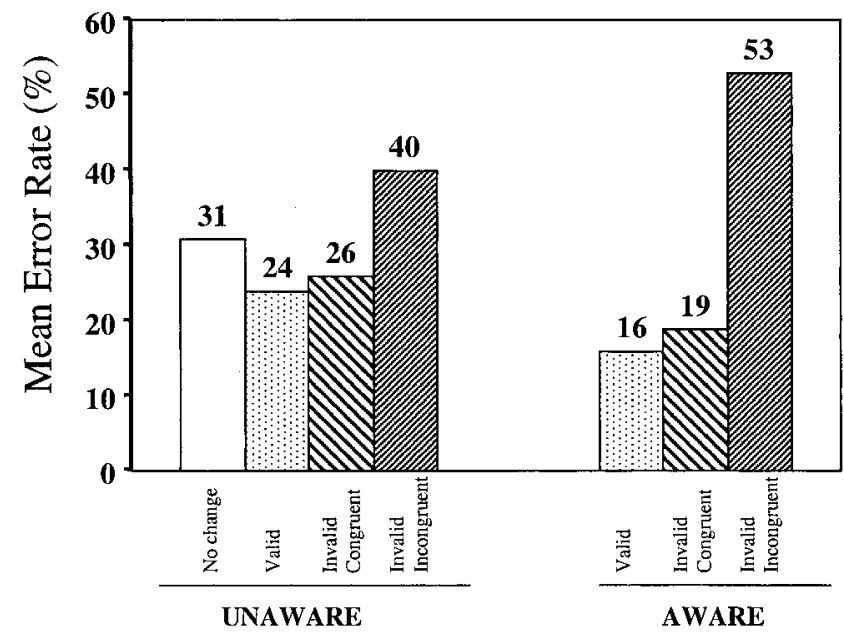

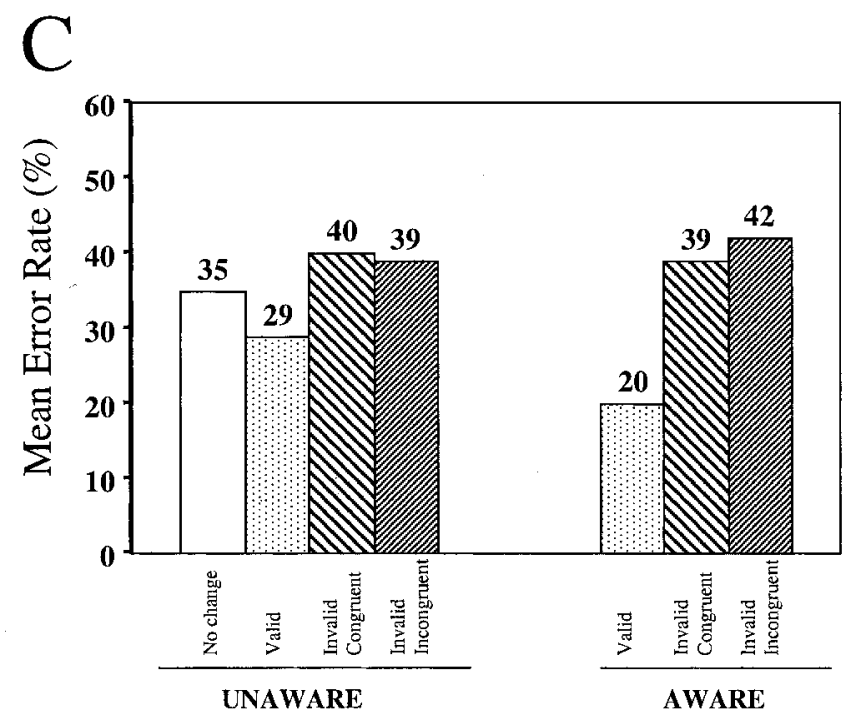

Figure 4. Data from Experiment 4. A: Results of the original study by Thornton and Fernandez-Duque (2000, Experiment 2). Adapted from "An Implicit Measure of Undetected Change," by I. M. Thornton \& D. Fernandez-Duque, 2000, Spatial Vision, 14, p. 36. Copyright 2000 by VSP. Adapted with permission. B and C: Results of Experiments 4A and 4B, respectively.

respond same and were sensitive to the change (mean $B^{\prime \prime}=.13$; mean $A^{\prime}=.82$ ).

Validity and awareness. As in Experiment 4A, this analysis compared performance on change trials for which the cued item was the changed item to performance on trials in which the cued item was not the changed item but was still congruent with the changed item (incongruent trials are not considered here). Observers generally made somewhat fewer errors when they were aware of the change, $F(1,19)=4.02, p=.059$, and they were more accurate on valid trials than on invalid trials, $F(1,19)=40.64, p<$ .001 ; see Table 3$)$. However, the interaction between awareness and validity was not significant, $F(1,19)=3.29, p=.085$. Even when observers were unaware of the change, they made fewer errors on valid trials $(M=29 \%)$ than on invalid congruent trials
$(M=40 \%), t(19)=2.83, p=.011$. We address whether or not this unexpected validity effect for unaware trials may reveal implicit change detection in the discussion of this experiment.

Congruence and awareness. As in Experiment 4A, this analysis compared performance on change trials for which the cue was congruent or incongruent with the changed item (excluding all valid trials). The critical analysis for the current hypothesis involved the congruence effect. If implicit identification underlies the congruence effect in Experiment 4A and in previous work (Thornton \& Fernandez-Duque, 2000), then the spatial decoupling of the changed item and the cued item should have little effect. In contrast, if the congruence effect depends on learning of the spatial contingency between the changed item and the cued item, then eliminating the predictive relationship should eliminate the con- 
Table 3

Mean Response Times (in Milliseconds) and Percentages of Error for Each Condition in Experiment $4 B$ as a Function of Awareness of the Change

\begin{tabular}{|c|c|c|c|c|}
\hline \multirow[b]{2}{*}{ Condition } & \multicolumn{2}{|c|}{ Response time (ms) } & \multicolumn{2}{|c|}{ Error rate $(\%)$} \\
\hline & Unaware & Aware & Unaware & Aware \\
\hline No change & $1,085(377)$ & $1,092(397)$ & $35.2(11)$ & $37.9(22)$ \\
\hline Valid & $1,132(579)$ & $1,034(404)$ & $28.7(20)$ & $19.9(14)$ \\
\hline Invalid congruent & $1,112(386)$ & $1,080(390)$ & $39.6(12)$ & $39.2(8)$ \\
\hline Invalid incongruent & $1,076(349)$ & $1,072(372)$ & $38.7(12)$ & $41.7(10)$ \\
\hline
\end{tabular}

Note. Standard deviations appear in parentheses.

gruence effect. Overall, in the absence of the predictive relationship, there was no main effect of awareness, $F(1,19)=0.77, p=$ .392. More important, there was no difference in accuracy for congruent and incongruent trials, $F(1,19)=0.34, p=.565$. Furthermore, congruence did not interact with awareness of the change, $F(1,19)=2.25, p=.150$. When observers were unaware of the change, the effect of congruence was not significant, $t(19)=$ $0.50, p=.621$ (see Figure 4).

\section{Discussion}

When the spatial relationship between the cue and the change locations was severed in Experiment 4B, observers no longer showed a congruence effect when they failed to detect the change. This finding suggests that the effects of the change identity on the orientation task in Experiment 4A resulted not from implicit identification but from the predictive spatial relationship between the cued item and the changed item. When asked, most of the observers in Experiment 4A reported noticing the spatial relationship. Given that observers became aware of this relationship and that the relationship was eliminated in Experiment 4B in both aware and unaware trials, we can infer that the effects shown in Experiment $4 \mathrm{~A}$ might have been explicit rather than implicit in origin. If the aware and unaware congruence effects are based on the same explicit factor (i.e., awareness of a spatial contingency), then both should disappear once the factor is eliminated.

Before it is concluded that no implicit identification occurred in these studies, two further issues must be considered. First, Experiment 4B might have lacked sufficient sensitivity to reveal implicit identification. The implicit identification process may be subtle, short-lived, and relatively weak. Consequently, it can be revealed only in the presence of additional spatial contingencies. However, the burden of proof for such a position rests with those wishing to posit an additional implicit mechanism when a single, plausible explicit mechanism can account for all of the results. Second, the persistence of the validity effect in Experiment $4 \mathrm{~B}$ on trials for which observers were unaware of the change might support the idea of implicit detection. Although Experiment 4B showed this effect, only one of the experiments in the original report (Thornton \& Fernandez-Duque, 2000, Experiment 2) showed the effect in error rates, and our Experiment 4A failed to find the effect. The results of our Experiment 1 might provide a potential explanation for the ephemeral nature of the validity effect. Differences in confidence may cause an effect of validity.

To examine the possibility that differences in confidence may cause a validity effect when observers report being unaware of the change, we tested a new group of 20 observers on a version of Experiment 4B in which we asked them to report their confidence in their change detection response after each trial. Mirroring Experiment $4 \mathrm{~B}$, when observers were unaware of the change, their error rates were no different on congruent $(M=41.96 \%, S D=$ $14.28 \%)$ and incongruent $(M=41.67 \%, S D=15.82 \%)$ trials, $t(19)=0.26, p=.802$. Thus, without a predictable spatial relationship between the cued item and the changed item, the congruence of the cued and changed items had no effect when observers were unaware of the change. The primary purpose of this additional experiment was to replicate the validity effect and then to determine whether or not it could be explained by confidence. However, we were unable to replicate the validity effect. When observers reported no awareness of the change, they showed no difference in error rates for valid trials $(M=40.32 \%, S D=$ $20.64 \%)$ and invalid congruent trials $(M=41.96 \%, S D=$ $14.28 \%), t(19)=0.72, p=.478$. Given the volatility of the validity effect across experiments, it is unclear whether this result is robust enough to merit a claim of implicit detection.

\section{GENERAL DISCUSSION}

Despite the alluring theoretical possibility of implicit change detection, we find that the existing empirical evidence for change detection can be explained through explicit comparison mechanisms. In four sets of experiments, we replicated the findings used to support claims of implicit detection and then showed, through both theoretical arguments and empirical analysis, that these results could be explained by explicit mechanisms. Although implicit change detection remains a theoretical possibility, the evidence to date does not provide compelling support for claims of implicit registration, localization, or identification.

Experiment 1 provided a potential explicit explanation for evidence of implicit registration: Differences in response times due to the presence of an unnoticed change might also result from differences in confidence combined with a bias to respond that there was no change (same). Note that it is possible that implicitly slowed response times actually affect rated confidence or that a third implicit factor causes both a reduction in confidence and a lengthening of response times. However, it is also plausible that confidence ratings reflect subcriterion explicit detection. Given this reasonable possibility, these findings do not provide incontrovertible evidence for implicit detection.

Experiments 2 and 3 raised theoretical and empirical objections to the evidence used to support implicit change localization. Experiment 2 showed that guessing of the change location was no 
better than would be expected on the basis of an explicit strategy of eliminating items known to be unchanged. This explicit strategy both explains previous evidence for better-than-chance guessing and predicts performance as exposure to the change is increased. Experiment 3 showed that previous claims regarding the implicit accumulation of information from the change location can be explained by differences in the detectability of changes within focused attention; the same pattern of results used to support claims of accumulation also emerges when all possibility of accumulation has been eliminated. Given that such results can also be explained by explicit search mechanisms, they fail to provide unequivocal support for implicit change detection. Finally, Experiment 4 suggested that the effects of the change identity on a secondary task can be explained by the spatial relationship between the changed item and the target of the secondary task. When that predictive relationship was eliminated, all implicit effects also disappeared. Given that the relationship was readily available and that it could guide explicit strategies, it is reasonable to infer that the effect could be driven by an explicit strategy rather than by an implicit comparison process.

All existing evidence is consistent with the claim that successful change detection, in the absence of a transient signal, requires an explicit comparison between a representation of the prechange scene and the postchange scene. That is, representations are not compared in the absence of an explicit comparison mechanism. This hypothesis is consistent with recent theoretical arguments that implicit mechanisms do not allow symbolic comparisons (Dulany, 1997). Change detection, by this account, occurs only as a result of an explicit comparison. Change blindness results when such comparisons fail, and in the face of change blindness, the change itself is not represented and can exert no influence on behavior. Note, of course, that a representation of the prechange scene could still exist and could influence behavior even if changes are not implicitly detected. In fact, findings of change blindness are entirely consistent with a preserved representation, provided that observers do not compare that representation to the changed scene (Simons, 2000). That is, change blindness might result from a comparison failure rather than a failure to represent certain aspects of a scene (e.g., Scott-Brown et al., 2000). Findings of change blindness, although suggestive, cannot provide evidence against the existence of representations of the changed scene.

If implicit comparisons do not contribute to change detection, how are changes detected in the absence of a change signal? Here we argue that changes are detected only through an explicit comparison of a prechange representation to the postchange scene (or the postchange representation if the scene is no longer available). Successful detection requires that attention be allocated to the location of the change both before and after the change occurs. Furthermore, observers must maintain attention on the change location long enough to form a representation of the features that changed. In a flicker task, change detection occurs as observers shift their attention from item to item, searching for the change. Attention is not drawn by the change itself. Rather, the properties of the individual scenes (e.g., areas of central interest or particularly salient regions; Rensink et al., 1997) might guide attention during this scan. The change itself does not constitute a signal for this attention guidance system, because no such signal exists in the absence of an explicit comparison between the original and the changed display. Change detection occurs not when the change draws attention but when observers happen to focus attention on the changing object, thereby instigating an explicit comparison. This conclusion is entirely consistent with evidence that change localization does not improve with repeated exposures to the change, provided that observers have not explicitly detected the change (Mitroff \& Simons, in press).

In a flicker task with simple displays, the assumption of an explicit, serial search mechanism predicts that the changing item will be located, on average, after half of the items have been attended. Provided that the changing item is no more salient than other items in either the original or changed display (e.g., if the changing item is embedded in an array of similar nonchanging items), detection should occur, on average, after the observer has attended to half of the items. Or, conversely, after half of the items have been inspected, the change should be detected $50 \%$ of the time. In fact, that is precisely what we found in the elimination condition of Experiment 2. When observers viewed the change for six cycles, they successfully eliminated an average of 4.11 of the 8 items (presumably by attending to those items). On average, they attended to approximately half of the items in the display, and consistent with the predictions of an explicit, serial search, they detected the change $50.01 \%$ of the time on these trials. If implicit detection were guiding the search process, performance should have been better than that predicted by an explicit, serial search.

Note that our strong hypothesis predicts the absence of an effect of implicit change detection. Detection should be no better than would be expected in the case of explicit comparison mechanisms. Given that we predict a null result, any incontrovertible evidence in support of implicit detection will refute our claim. If, as some have argued, implicit comparison mechanisms are not possible in principle, then a close examination of any claim for implicit detection should reveal some degree of explicit contamination. We have provided several examples of the influence that explicit strategies can have on change detection even when observers report no awareness of changes. A variety of approaches might eventually produce evidence of implicit detection. For example, event-related potential or functional magnetic resonance imaging evidence could well provide a more sensitive measure to dissociate implicit change detection from explicit strategies. However, as with the strictly behavioral measures, researchers need to take care to avoid subtle explicit influences. Furthermore, they must avoid conflating evidence for a representation of the prechange object with evidence for an implicit comparison of the original and the changed object. Implicit change detection is an alluring theoretical possibility and one that should receive full empirical examination. However, the often subtle but significant role played by explicitly available contingencies in these change detection tasks serves as a caution against the "siren song" of implicit change detection.

\section{References}

Angelone, B. L., Levin, D. T., \& Simons, D. J. (2001). Representation and comparison failures in change blindness. Manuscript submitted for publication.

Butler, L. T., \& Berry, D. C. (2001). Implicit memory: Intention and awareness revisited. Trends in Cognitive Sciences, 5, 192-197.

Cavanagh, P., MacLeod, D. I. A., \& Anstis, S. M. (1987). Equiluminance: Spatial and temporal factors and the contribution of blue-sensitive cones. Journal of the Optical Society of America, 4(A), 1428-1438.

Chun, M. M., \& Jiang, Y. (1998). Contextual cueing: Implicit learning and memory of visual context guides spatial attention. Cognitive Psychology, $36,28-71$ 
Chun, M. M., \& Nakayama, K. (2000). On the functional role of implicit visual memory for the adaptive deployment of attention across scenes. Visual Cognition, 7, 65-82.

Dixon, N. F. (1981). Preconscious processing. New York: Wiley.

Dulany, D. E. (1997). Consciousness in the explicit (deliberative) and implicit (evocative). In J. D. Cohen \& J. W. Schooler (Eds.), Scientific approaches to consciousness (pp. 179-211). Mahwah, NJ: Erlbaum.

Fernandez-Duque, D., \& Thornton, I. M. (2000). Change detection without awareness: Do explicit reports underestimate the representation of change in the visual system? Visual Cognition, 7, 323-344.

Grimes, J. (1996). On the failure to detect changes in scenes across saccades. In K. Akins (Ed.), Vancouver studies in cognitive science: Perception (Vol. 2, pp. 89-110). New York: Oxford University Press.

Hayhoe, M. M., Bensinger, D. G., \& Ballard, D. (1998). Task constraints in visual working memory. Vision Research, 38, 125-137.

Henderson, J. M., \& Hollingworth, A. (1999). The role of fixation position in detecting scene changes across saccades. Psychological Science, 10, $438-443$.

Holender, D. (1986). Semantic activation without conscious identification in dichotic listening, parafoveal vision, and visual masking: A survey and appraisal. Behavioral and Brain Sciences, 9, 1-66.

Hollingworth, A., Williams, C. C., \& Henderson, J. M. (2001). To see and remember: Visually specific information is retained in memory from previously attended objects in natural scenes. Psychonomic Bulletin \& Review, 8, 761-768.

Horowitz, T. S., \& Wolfe, J. M. (1998, August 6). Visual search has no memory. Nature, 394, 575-577.

James, W. (1950). The principles of psychology (Vol. 1). New York: Dover. (Original work published 1891)

Levin, D. T., \& Simons, D. J. (1997). Failure to detect changes to attended objects in motion pictures. Psychonomic Bulletin \& Review, 4, 501-506.

Maljkovic, V., \& Nakayama, K. (1994). Priming of pop-out: I. Role of features. Memory \& Cognition, 22, 657-672.

Maljkovic, V., \& Nakayama, K. (1996). Priming of pop-out: II. The role of position. Perception \& Psychophysics, 58, 977-991.

Merikle, P. M., \& Reingold, E. M. (1998). On demonstrating unconscious perception: Comment on Draine and Greenwald (1998). Journal of Experimental Psychology: General, 127, 304-310.

Mitroff, S. R., \& Simons, D. J. (in press). Changes are not localized before they are explicitly detected. Visual Cognition.

O'Regan, J. K., Deubel, H., Clark, J. J., \& Rensink, R. A. (1997). Picture changes during blinks: Not seeing where you look and seeing where you don't look. Investigative Ophthalmology \& Visual Science, 38, S707.

O’Regan, J. K., Rensink, R. A., \& Clark, J. J. (1999, March 4). Changeblindness as a result of "mudsplashes." Nature, 398, 34.

Pashler, H. (1988). Familiarity and visual change detection. Perception \& Psychophysics, 44, 369-378.

Ratcliff, R., \& McKoon, G. (1995). Bias in the priming of object decisions.
Journal of Experimental Psychology: Learning, Memory, and Cognition, 21, 754-767.

Reingold, E. M., \& Merikle, P. M. (1988). Using direct and indirect measures to study perception without awareness. Perception \& Psychophysics, 44, 563-575.

Rensink, R. A. (2000a). Seeing, sensing, and scrutinizing. Vision Research, 40, 1469-1487.

Rensink, R. A. (2000b). Visual search for change: A probe into the nature of attentional processing. Visual Cognition, 7, 345-376.

Rensink, R. A., O’Regan, J. K., \& Clark, J. J. (1997). To see or not to see: The need for attention to perceive changes in scenes. Psychological Science, 8, 368-373.

Rensink, R. A., O’Regan, J. K., \& Clark, J. J. (2000). On the failure to detect changes in scenes across brief interruptions. Visual Cognition, 7, $127-146$.

Rosenthal, R., \& Rosnow, R. L. (1991). Essentials of behavioral research: Methods and data analysis (2nd ed.). New York: McGraw-Hill.

Scott-Brown, K. C., Baker, M. R., \& Orbach, H. S. (2000). Comparison blindness. Visual Cognition, 7, 253-267.

Shore, D., \& Klein, R. M. (2000). The effects of scene inversion on change blindness. Journal of General Psychology, 127, 27-43.

Simons, D. J. (1996). In sight, out of mind: When object representations fail. Psychological Science, 7, 301-305.

Simons, D. J. (2000). Current approaches to change blindness. Visual Cognition, 7, 1-15.

Simons, D. J., Chabris, C. F., Schnur, T., \& Levin, D. T. (2002). Evidence for preserved representations in change blindness. Consciousness and Cognition, 11, 78-97.

Simons, D. J., Franconeri, S. L., \& Reimer, R. L. (2000). Change blindness in the absence of a visual disruption. Perception, 29, 1143-1154.

Simons, D. J., \& Levin, D. T. (1997). Change blindness. Trends in Cognitive Sciences, 1, 261-267.

Simons, D. J., \& Levin, D. T. (1998). Failure to detect changes to people in a real-world interaction. Psychonomic Bulletin \& Review, 5, $644-$ 649.

Smilek, D., Eastwood, J. D., \& Merikle, P. M. (2000). Does unattended information facilitate change detection? Journal of Experimental Psychology: Human Perception and Performance, 26, 480-487.

Snodgrass, J. G., \& Corwin, J. (1988). Pragmatics of measuring recognition memory: Applications to dementia and amnesia. Journal of Experimental Psychology: General, 117, 34-50.

Thornton, I. M., \& Fernandez-Duque, D. (2000). An implicit measure of undetected change. Spatial Vision, 14, 21-44.

Williams, P., \& Simons, D. J. (2000). Detecting changes in novel, complex three-dimensional objects. Visual Cognition, 7, 297-322.

Received April 23, 2001

Revision received October 25, 2001

Accepted November 8, 2001 